\title{
The non-uniqueness of the atomistic stress tensor and its relationship to the generalized Beltrami representation
}

\author{
Nikhil Chandra Admal and E. B. Tadmor \\ Department of Aerospace Engineering and Mechanics \\ University of Minnesota, Minneapolis, MN 55455, USA
}

\begin{abstract}
The non-uniqueness of the atomistic stress tensor is a well-known issue when defining continuum fields for atomistic systems. In this paper, we study the non-uniqueness of the atomistic stress tensor stemming from the non-uniqueness of the potential energy representation. In particular, we show using rigidity theory that the distribution associated with the potential part of the atomistic stress tensor can be decomposed into an irrotational part that is independent of the potential energy representation, and a traction-free solenoidal part. Therefore, we have identified for the atomistic stress tensor a discrete analog of the continuum generalized Beltrami representation (a version of the vector Helmholtz decomposition for symmetric tensors). We demonstrate the validity of these analogies using a numerical test. A program for performing the decomposition of the atomistic stress tensor called MDStressLab is available online at http://mdstresslab.org.
\end{abstract}

Keywords: A.stress relaxation, A.stress concentration, B.constitutive behavior, B.residual stress, C.atomistics

\section{Introduction}

Atomistic simulations are extensively used as a tool to predict constitutive relations for continuum models. In order to do so, the data generated in an atomistic simulation, which is in the form of positions and velocities of particles, has to be reinterpreted in the language of continuum mechanics. This is done by developing continuum notions for atomistic systems. Work on this dates back at least to Cauchy in the 1820 s with his aim to define stress in crystalline solids. Later, Irving and Kirkwood (1950) derived continuum fields for an atomistic system that is probabilistic in nature using the principles of non-equilibrium classical statistical mechanics. More recently, Hardy (1982); Hardy et al. (2002) and Murdoch (1983) defined continuum fields for an atomistic model using spatial averaging.

Of all continuum fields, the atomistic stress tensor has been studied most extensively due to its non-uniqueness. The most commonly used atomistic stress definitions are the virial (Maxwell, 1870, 1874), Tsai (Tsai, 1979), Irving-Kirkwood (Irving and Kirkwood, 1950), and MurdochHardy (Murdoch, 2003) stress tensors. In recent work of Admal and Tadmor (2010, 2011), the authors extensively studied the definition for the stress tensor within a unified framework based on a generalization of the Irving-Kirkwood procedure to arbitrary multi-body potentials. Through this unified framework it is shown that all existing definitions follow as special cases 
of a single stress expression. One of the sources of non-uniqueness of the atomistic stress tensor is identified to be the non-uniqueness of force decomposition, which is in turn related to the non-uniqueness of the potential energy representation used in the calculation. The term "representation" reflects the fact that the potential energy, which is a function of distances between atoms, must be extended in a non-unique fashion off the subspace corresponding to real physical distances in order to compute the derivatives appearing in the stress expression.

In the absence of a sound quantum mechanical origin for potential energy representations, we focus our attention on characterizing the non-uniqueness of the force decomposition. Our analysis is based on rigidity theory, which results in a decomposition of the distribution associated with the potential part of the atomistic stress tensor in the Murdoch-Hardy procedure into an irrotational part that is independent of the potential energy representation, and a traction-free solenoidal part. This decomposition has a potential application in systematic reduction of noise in atomistic fields. A similar decomposition of the Cauchy stress tensor can be constructed in continuum mechanics using the generalized Beltrami representation, which is an analogy for symmetric tensors of the vector Helmholtz decomposition. We identify analogies between the two decompositions, and in the process, obtain a strain tensor equivalent in atomistics. Finally, we compare the two decompositions through a numerical test, and demonstrate their equivalence. In other words, we have obtained a discrete version of the Beltrami representation for the atomistic stress tensor. ${ }^{1}$

The paper is organized as follows. In Section 2, we review the derivation of the atomistic stress tensor in the Murdoch-Hardy procedure. In Section 3, we identify the non-uniqueness of the force decomposition that results in the non-uniqueness of atomistic the stress tensor, and give it a geometric interpretation. In Section 4, we decompose the potential part of the atomistic stress tensor into a solenoidal part and an irrotational part, and identify the properties of the decomposition. In Section 5, we review the generalized Beltrami representation for symmetric tensor fields, and obtain an orthogonal decomposition of a symmetric tensor field into a tractionfree solenoidal part and an irrotational part. In Section 7, we demonstrate the equivalence of the decompositions proposed in Sections 4 and 5 using a numerical test. We conclude with a summary in Section 8.

\subsection{Notation}

In this paper, we use lower case bold letters to denote vectors, and upper case bold letters to denote second-order tensors. The gradient, divergence and curl operators are denoted by the symbols $\nabla$, div and curl respectively. We use the symbol $\otimes$ to denote the tensor product of two vectors, and $\cdot$ to denote the inner product of two vectors or tensors.

\section{The atomistic stress tensor in the Murdoch-Hardy procedure}

An atomistic system is modeled as a collection of $N$ interacting point particles in the Euclidean ${ }^{2}$ space $\mathbb{R}^{3}$. The positions and velocities of the point particles at time $t$ are given by the

\footnotetext{
${ }^{1}$ Discrete versions of the Beltrami representation for symmetric tensors are often discussed in the finite elements literature (for example, see Hauret et al. (2007)) in the context of constructing discrete operators on the finite element mesh to preserve the symplectic geometry of the system. To our knowledge, no such representation exists for atomistic systems prior to this work.

${ }^{2}$ All real coordinate vector spaces appearing in this paper are equipped with the standard inner product.
} 
functions $\boldsymbol{x}_{\alpha}(t)$ and $\boldsymbol{v}_{\alpha}(t)(\alpha=1, \ldots, N, t \geq 0)$, respectively. We also use the expressions $\boldsymbol{x}_{\alpha}$ and $\boldsymbol{v}_{\alpha}$ to denote the value of the functions $\boldsymbol{x}_{\alpha}(\cdot)$ and $\boldsymbol{v}_{\alpha}(\cdot)$ at a particular instant of time. The system is assumed to be conservative, which means that there exists a potential energy function $\widehat{\mathcal{V}}\left(\boldsymbol{x}_{1}, \boldsymbol{x}_{2}, \ldots, \boldsymbol{x}_{N}\right)$ for the interaction of the particles. This function is taken to be differentiable, so that the force on particle $\alpha$ follows as the negative gradient of the potential energy with respect to its position,

$$
\boldsymbol{f}_{\alpha}=-\nabla_{\boldsymbol{x}_{\alpha}} \widehat{\mathcal{V}}
$$

We assume a finite range of interaction for $\widehat{\mathcal{V}}$. This means that there exists a cutoff radius $r_{\text {cut }}>0$ such that $\widehat{\mathcal{V}}$ can be decomposed as a finite sum of particle potential energies, ${ }^{3}$

$$
\widehat{\mathcal{V}}=\sum_{\alpha=1}^{M} \widehat{\mathcal{V}}_{\alpha}
$$

where each $\widehat{\mathcal{V}}_{\alpha}$ in Eqn. (2.2) is a conservative potential function of the positions of particles within a cluster of size $r_{\text {cut }}$. No restrictions are placed on the form of $\widehat{\mathcal{V}}_{\alpha}$, which can be a general many-body potential.

The atomistic stress can be derived using the Murdoch-Hardy ( $\mathrm{MH}$ ) or Irving-Kirkwood (IK) procedures. For the purpose of this paper, we discuss the atomistic stress tensor and its uniqueness using the $\mathrm{MH}$ procedure, although a similar description can be carried out using IK. Under the MH procedure, continuum fields are defined as direct spatial averages of microscopic variables. The spacial averaging is performed using a weighting function $w: \mathbb{R}^{3} \rightarrow \mathbb{R}^{+}$with compact support. The weighting function must satisfy the normalization condition

$$
\int_{\mathbb{R}^{3}} w(\boldsymbol{r}) d \boldsymbol{r}=1
$$

The derivation of the atomistic stress begins with the definition of the mass density, momentum density, and velocity fields:

$$
\begin{aligned}
\rho_{w}(\boldsymbol{x}, t) & :=\sum_{\alpha} m_{\alpha} w\left(\boldsymbol{x}_{\alpha}-\boldsymbol{x}\right) \\
\boldsymbol{p}_{w}(\boldsymbol{x}, t) & :=\sum_{\alpha} m_{\alpha} \boldsymbol{v}_{\alpha} w\left(\boldsymbol{x}_{\alpha}-\boldsymbol{x}\right) \\
\boldsymbol{v}_{w}(\boldsymbol{x}, t) & :=\boldsymbol{p}_{w} / \rho_{w}
\end{aligned}
$$

where $\boldsymbol{x} \in \mathbb{R}^{3}$, and the subscript $w$ indicates the role of the weighting function. In order to arrive at an expression for the atomistic stress tensor, denoted by $\boldsymbol{\sigma}_{w}$, it is postulated that the fields $\rho_{w}, \boldsymbol{v}_{w}$ and $\boldsymbol{\sigma}_{w}$, satisfy the equation of motion from continuum mechanics (see e.g. Tadmor et al. (2012)). For simplicity, we assume that there are no body forces acting on the system. We

\footnotetext{
${ }^{3}$ Note that the decomposition given in Eqn. (2.2) is not unique. No physical meaning is attached to the particle energies $\widehat{\mathcal{V}}_{\alpha}$. For more on the non-uniqueness of the energy decomposition, see Admal and Tadmor (2011).
} 
therefore have

$$
\frac{\partial\left(\rho_{w} \boldsymbol{v}_{w}\right)}{\partial t}+\operatorname{div}_{\boldsymbol{x}}\left(\rho_{w} \boldsymbol{v}_{w} \otimes \boldsymbol{v}_{w}\right)=\operatorname{div}_{\boldsymbol{x}} \boldsymbol{\sigma}_{w}
$$

Substituting the definitions for $\rho_{w}$ and $\boldsymbol{v}_{w}$ given in Eqn. (2.4) into Eqn. (2.5), with some additional algebra (see Admal and Tadmor (2010) for the full derivation), we arrive at an expression for the divergence of the atomistic stress tensor given by

$$
\operatorname{div}_{\boldsymbol{x}} \boldsymbol{\sigma}_{w}(\boldsymbol{x}, t)=\sum_{\alpha} \boldsymbol{f}_{\alpha}(t) w\left(\boldsymbol{x}_{\alpha}(t)-\boldsymbol{x}\right)-\operatorname{div}_{\boldsymbol{x}} \sum_{\alpha} m_{\alpha}\left(\boldsymbol{v}_{\alpha}^{\mathrm{rel}} \otimes \boldsymbol{v}_{\alpha}^{\mathrm{rel}}\right) w\left(\boldsymbol{x}_{\alpha}-\boldsymbol{x}\right),
$$

where $\boldsymbol{f}_{\alpha}(t)$ is defined in Eqn. (2.1), and $\boldsymbol{v}_{\alpha}^{\mathrm{rel}}(\boldsymbol{x}, t):=\boldsymbol{v}_{\alpha}(t)-\boldsymbol{v}_{w}(\boldsymbol{x}, t)$. From Eqn. (2.6), it is clear that the stress tensor has two contributions, commonly referred to as the potential and kinetic parts of the stress tensor. The kinetic part, denoted by $\boldsymbol{\sigma}_{w, \mathrm{k}}$, is given by

$$
\boldsymbol{\sigma}_{w, \mathrm{k}}(\boldsymbol{x}, t)=-\sum_{\alpha} m_{\alpha}\left(\boldsymbol{v}_{\alpha}^{\mathrm{rel}} \otimes \boldsymbol{v}_{\alpha}^{\mathrm{rel}}\right) w\left(\boldsymbol{x}_{\alpha}-\boldsymbol{x}\right),
$$

and the potential part of the stress tensor, denoted by $\boldsymbol{\sigma}_{w, \mathrm{v}}$, satisfies the equation

$$
\operatorname{div}_{\boldsymbol{x}} \boldsymbol{\sigma}_{w, \mathrm{v}}(\boldsymbol{x}, t)=\sum_{\alpha} \boldsymbol{f}_{\alpha}(t) w\left(\boldsymbol{x}_{\alpha}(t)-\boldsymbol{x}\right) .
$$

It is clear that there are many candidates for $\boldsymbol{\sigma}_{w, \mathrm{v}}$ that satisfy Eqn. (2.8). Examples include the Hardy (Hardy, 1982), Murdoch (Murdoch, 2007) and the Tsai (Tsai, 1979) stress tensors. The procedure to arrive at these stress tensors involves the following three steps:

1. The force $\boldsymbol{f}_{\alpha}$ in Eqn. (2.8) is decomposed as

$$
\boldsymbol{f}_{\alpha}=\sum_{\substack{\beta \\ \beta \neq \alpha}} \boldsymbol{f}_{\alpha \beta}
$$

where $\boldsymbol{f}_{\alpha \beta}=-\boldsymbol{f}_{\beta \alpha}$. We call a decomposition central force if $\boldsymbol{f}_{\alpha \beta}$ is parallel to the line joining particles $\alpha$ and $\beta$, and non-central force otherwise. Substituting Eqn. (2.9) into Eqn. (2.8), we obtain ${ }^{4}$

$$
\operatorname{div}_{\boldsymbol{x}} \boldsymbol{\sigma}_{w, \mathrm{v}}(\boldsymbol{x}, t)=\sum_{\substack{\alpha, \beta \\ \alpha \neq \beta}} \boldsymbol{f}_{\alpha \beta} w\left(\boldsymbol{x}_{\alpha}(t)-\boldsymbol{x}\right) .
$$

2. The sum in Eqn. (2.10) is written as an integral of a generating function $\boldsymbol{g}(\boldsymbol{x}, \boldsymbol{y})$ that is anti-symmetric in its arguments, with certain decay properties (Admal and Tadmor, 2010):

$$
\operatorname{div}_{\boldsymbol{x}} \boldsymbol{\sigma}_{w, \mathrm{v}}(\boldsymbol{x}, t)=\int_{\mathbb{R}^{3}} \boldsymbol{g}(\boldsymbol{x}, \boldsymbol{y}, t) d \boldsymbol{y}
$$

\footnotetext{
${ }^{4}$ The following notation is used: $\sum_{\substack{\alpha, \beta \\ \alpha \neq \beta}}=\sum_{\alpha} \sum_{\substack{\beta \neq \alpha \\ \beta}}$.
} 
Different choices of the generating function result in different stress tensors. For instance, the generating function for the Hardy stress is given by

$$
\boldsymbol{g}^{\mathrm{H}}(\boldsymbol{x}, \boldsymbol{y}, t)=\sum_{\substack{\alpha, \beta \\ \alpha \neq \beta}} \boldsymbol{f}_{\alpha \beta} w\left(\boldsymbol{x}_{\alpha}-\boldsymbol{x}\right) \delta\left(\boldsymbol{x}_{\beta}-\boldsymbol{x}_{\alpha}+\boldsymbol{x}-\boldsymbol{y}\right)
$$

where $\delta$ is the Dirac delta distribution in $\mathbb{R}^{3}$.

3. Finally, Noll's lemma (Noll, 1955) is invoked to express the right-hand-side of Eqn. (2.11) in divergence form, from which an expression for the stress tensor follows. The stress tensor proposed by Hardy (1982); Hardy et al. (2002) is obtained using the kernel $\boldsymbol{g}^{\mathrm{H}}$ :

$$
\boldsymbol{\sigma}_{w, \mathrm{v}}=\frac{1}{2} \sum_{\substack{\alpha, \beta \\ \alpha \neq \beta}} \int_{s=0}^{1}\left[-\boldsymbol{f}_{\alpha \beta} w\left((1-s) \boldsymbol{x}_{\alpha}+s \boldsymbol{x}_{\beta}-\boldsymbol{x}\right) \otimes\left(\boldsymbol{x}_{\alpha}-\boldsymbol{x}_{\beta}\right)\right] d s .
$$

If $\boldsymbol{f}_{\alpha \beta}$ is parallel to the line joining particles $\alpha$ and $\beta$, the Hardy stress $\boldsymbol{\sigma}_{w, \mathrm{v}}$ is symmetric.

We note that the fields $\rho_{w}, \boldsymbol{p}_{w}$, and $\boldsymbol{\sigma}_{w, \mathrm{v}}$ obtained in the MH procedure can be written as a convolution $^{5}$ of the weighting function $w$ with a corresponding distribution ${ }^{6}$. In other words,

$$
\rho_{w}(\boldsymbol{x}, t)=w \star \rho, \quad \boldsymbol{p}_{w}(\boldsymbol{x}, t)=w \star \boldsymbol{p}, \quad \boldsymbol{\sigma}_{w, \mathrm{v}}(\boldsymbol{x}, t)=w \star \boldsymbol{\sigma}_{\mathrm{v}},
$$

where $\star$ denotes the convolution operator, and the distributions $\rho, \boldsymbol{p}$, and $\boldsymbol{\sigma}_{\mathrm{v}}$ are given by

$$
\begin{aligned}
\rho(\boldsymbol{x}, t) & =\sum_{\alpha} m_{\alpha} \delta\left(\boldsymbol{x}_{\alpha}-\boldsymbol{x}\right), \\
\boldsymbol{p}(\boldsymbol{x}, t) & =\sum_{\alpha} m_{\alpha} \boldsymbol{v}_{\alpha} \delta\left(\boldsymbol{x}_{\alpha}-\boldsymbol{x}\right) \\
\boldsymbol{\sigma}_{\mathrm{v}}(\boldsymbol{x}, t) & =\sum_{\substack{\alpha, \beta \\
\alpha<\beta}}-\boldsymbol{f}_{\alpha \beta} \otimes\left(\boldsymbol{x}_{\alpha}-\boldsymbol{x}_{\beta}\right) \int_{s=0}^{1} \delta\left((1-s) \boldsymbol{x}_{\alpha}+s \boldsymbol{x}_{\beta}-\boldsymbol{x}\right) d s .
\end{aligned}
$$

By writing the spatially-averaged fields $\rho_{w}, \boldsymbol{p}_{w}$ and $\boldsymbol{\sigma}_{w, \mathrm{v}}$ in convolution form, we are able to separate out the role of the weighting function, and thereby arrive at distributions $\rho, \boldsymbol{p}$ and $\boldsymbol{\sigma}_{\mathrm{v}}$ that are independent of it.

Let us now discuss the various sources of non-uniqueness of the potential part of the atomistic stress tensor $\boldsymbol{\sigma}_{w, \mathrm{v}}=w \star \boldsymbol{\sigma}_{\mathrm{v}}$ resulting from the $\mathrm{MH}$ procedure described above. The first source of non-uniqueness is the choice of the weighting function $w$. See for example Ulz et al. (2013)

\footnotetext{
${ }^{5}$ See Hörmander (1990) for the formal definition of a convolution of a function with a distribution. We do not require such a general definition. This is because the distributions $\rho, \boldsymbol{p}$ and $\boldsymbol{\sigma}_{\mathrm{v}}$, defined in Eqns. (2.15), (2.16) and (2.17) respectively, are derived from a Dirac delta distribution on which algebra can be carried out as if it were a function. Therefore, we use the following definition for the convolution of two smooth functions $u$ and $v$ that have a compact support in $\mathbb{R}^{3}$

$$
u \star v(\boldsymbol{x}):=\int_{\mathbb{R}^{3}} u(\boldsymbol{x}-\boldsymbol{y}) v(\boldsymbol{y}) d \boldsymbol{y}
$$

where $v$ is replaced by distributions $\rho, \boldsymbol{p}$ or $\boldsymbol{\sigma}_{\mathrm{v}}$.

${ }^{6} \mathrm{~A}$ distribution is a linear map from the space of smooth functions with compact support to the real line $\mathbb{R}$.
} 
for a discussion on the optimal choice of $w$ based on the correlation length of the potential energy function. The second source of non-uniqueness is the choice of the force decomposition mentioned above in step 1, which affects $\sigma_{\mathrm{v}}$ (Admal and Tadmor, 2010, 2011). A recent article by Vanegas et al. (2014) discusses the significance of the force decomposition on the atomistic stress in lipid bilayers used to evaluate the material constants of the bilayer membrane. The third source of non-uniqueness is the choice of the kernel function mentioned above in step 2. See Admal and Tadmor (2010) for kernel functions that result in various atomistic stress tensors.

The main focus of this paper is the non-uniqueness of $\sigma_{\mathrm{v}}$ resulting due to the non-uniqueness of the force decomposition and the derivation of a decomposition that separates $\sigma_{\mathrm{v}}$ into unique and non-unique parts. In the next section, we present some general properties of the decomposition given in Eqn. (2.9), demonstrate its non-uniqueness, and set up a discussion for its interpretation.

\section{Non-uniqueness of the force decomposition}

It is clear that the force decomposition in Eqn. (2.9) represents an over-determined system of $3 N$ equations with $3 N(N-1)$ unknowns. ${ }^{7}$ This implies that there are can be many solutions for $f_{\alpha \beta}$. Thus in order to arrive at a reasonable force decomposition, additional conditions are required. Two such conditions are obtained if we interpret $f_{\alpha \beta}$ as the force on particle $\alpha$ due to the presence of particle $\beta$, and enforce the condition of momentum balance and angular momentum balance for any subset of particles within the body. These conditions are referred to in Admal and Tadmor (2010) as the "strong" and "weak law of action and reaction" respectively, and are given by

$$
\begin{aligned}
& \boldsymbol{f}_{\alpha \beta}=-\boldsymbol{f}_{\beta \alpha} \\
& \boldsymbol{f}_{\alpha \beta} \| \boldsymbol{x}_{\alpha}-\boldsymbol{x}_{\beta} .
\end{aligned}
$$

Equations (3.1) and (3.2) reduce the number of unknowns in Eqn. (2.9) from $3 N(N-1)$ to $N(N-1) / 2$, where the unknowns are now represented as a set of scalars $\left\{f_{\alpha \beta}: \alpha, \beta=\right.$ $1, \ldots, N$, and $\alpha \neq \beta\}$. Note that for $N>4$, in spite of the reduction in the number of unknowns, the system of equations remains over-determined. We will now give a geometric interpretation of the non-uniqueness of a force decomposition satisfying Eqns. (3.1) and (3.2). In order to do so, we will need the following definitions.

An edge is defined as a two-element subset $\{\alpha, \beta\}$ of $\{1, \ldots, N\}$. We use the shorthand notation $\alpha \beta$ to denote an edge. Let $E$ denote the ordered set of all edges, with a strict order given by

$$
\alpha \beta<\gamma \delta \Longleftrightarrow\left\{\begin{array}{l}
\min (\alpha, \beta)<\min (\gamma, \delta) \text { or } \\
\min (\alpha, \beta)=\min (\gamma, \delta), \max (\alpha, \beta)<\max (\gamma, \delta)
\end{array}\right.
$$

In other words, the edges are ordered as $\{1,2\}<\{1,3\}<\cdots<\{1, N\}<\{2,3\}<\cdots<$ $\{N-1, N\}$. Let $\boldsymbol{x}=\left(\boldsymbol{x}_{1}, \ldots, \boldsymbol{x}_{N}\right) \in \mathbb{R}^{3 N}$ denote the configuration of interest, and $E_{\boldsymbol{x}}$ denote

\footnotetext{
${ }^{7}$ There are $N(N-1)$ forces since in general $\boldsymbol{f}_{\alpha \beta}$ can be different from $\boldsymbol{f}_{\beta \alpha}$. The factor 3 is included because force is a 3 -dimensional vector
} 
the ordered subset of edges in $E$ falling within the range of interaction,

$$
E_{\boldsymbol{x}}:=\left\{\alpha \beta:\left\|\boldsymbol{x}_{\alpha}-\boldsymbol{x}_{\beta}\right\|<r_{\text {cut }}\right\} .
$$

In particular, let $E_{\boldsymbol{x}}(k)$ denote the $k$-th edge in $E_{\boldsymbol{x}}$. Denote the cardinality (i.e. number of elements) of $E_{\boldsymbol{x}}$ as $e$. It can be easily shown that there exists a neighborhood $\mathcal{N}_{\boldsymbol{x}}$ of $\boldsymbol{x}$ in $\mathbb{R}^{3 N}$ such that for every $\boldsymbol{y} \in \mathcal{N}_{\boldsymbol{x}}, E_{\boldsymbol{y}}=E_{\boldsymbol{x}}$. In other words, for every configuration $\boldsymbol{y} \in \mathcal{N}_{\boldsymbol{x}}$, the lengths of edges present in $E_{\boldsymbol{x}}$ and its complement, remain less than and greater than $r_{\text {cut }}$, respectively. We refer to $\mathcal{N}_{\boldsymbol{x}}$ as the local configuration space at $\boldsymbol{x}$.

The local configuration space can be described in terms of "local distances" using a local rigidity map ${ }^{8} \boldsymbol{R}: \mathcal{N}_{\boldsymbol{x}} \rightarrow \mathbb{R}^{e}$ defined as

$$
\boldsymbol{R}(\boldsymbol{y})=\left(r_{E_{\boldsymbol{x}}(1)}, \ldots, r_{E_{\boldsymbol{x}}(e)}\right), \quad \boldsymbol{y} \in \mathcal{N}_{\boldsymbol{x}},
$$

with $r_{E_{\boldsymbol{x}}}(k)=\left\|\boldsymbol{y}_{\alpha}-\boldsymbol{y}_{\beta}\right\|$, where $E_{\boldsymbol{x}}(k)=\alpha \beta$. We define the local shape space $\mathcal{S}_{\boldsymbol{x}}$ as the image ${ }^{9}$ of the local rigidity map:

$$
\mathcal{S}_{x}:=\operatorname{Im} \boldsymbol{R} .
$$

The term "local" is being used because each $e$-tuple in $\mathcal{S}_{x}$ contains only those distances that are less than $r_{\text {cut }}$, as opposed to the shape space defined in Admal and Tadmor (2010) which includes all distances. We adopt the definition in Eqn. (3.6) to make use of the finite range of interaction assumption implicit in Eqn. (2.2), which will be useful in our study of the force decomposition in Section 4. In addition, we note that since the local distances are not independent of each other, the local shape space may be viewed as a lower-dimensional surface in $\mathbb{R}^{e}$. It can be shown that each $\widehat{\mathcal{V}}_{\alpha}$ in Eqn. (2.2), viewed as a function on $\mathbb{R}^{3 N}$, and restricted to $\mathcal{N}_{\boldsymbol{x}}$, can be represented uniquely ${ }^{10}$ by a function $\breve{\mathcal{V}}_{\alpha}: \mathcal{S}_{x} \rightarrow \mathbb{R}$. In other words,

$$
\widehat{\mathcal{V}}_{\alpha}(\boldsymbol{y})=\breve{\mathcal{V}}(\boldsymbol{R}(\boldsymbol{y})), \quad \forall \boldsymbol{y} \in \mathcal{N}_{\boldsymbol{x}} .
$$

Therefore, from Eqn. (2.2) it follows that $\widehat{\mathcal{V}}$ restricted to $\mathcal{N}_{\boldsymbol{x}}$ can be represented as a new func$\operatorname{tion}^{11}, \breve{\mathcal{V}}:=\sum_{\alpha} \breve{\mathcal{V}}_{\alpha}: \mathcal{S}_{\boldsymbol{x}} \rightarrow \mathbb{R}$

In order to obtain a force decomposition, we now assume that there exists an extension $\mathcal{V}$ of the function $\breve{\mathcal{V}}$ defined on $\mathcal{S}_{\boldsymbol{x}}$ to a neighborhood of $\mathcal{S}_{\boldsymbol{x}} \in \mathbb{R}^{e}$, i.e

$$
\left.\mathcal{V}\right|_{\mathcal{S}_{x}} \equiv \breve{\mathcal{V}}
$$

and define the force $\boldsymbol{f}_{\alpha \beta}$ for any configuration $\boldsymbol{y} \in \mathcal{N}_{\boldsymbol{x}}$ as

$$
\boldsymbol{f}_{\alpha \beta}:= \begin{cases}-\frac{\partial \mathcal{V}}{\partial r_{\alpha \beta}}(\boldsymbol{R}(\boldsymbol{y})) \frac{\boldsymbol{y}_{\alpha}-\boldsymbol{y}_{\beta}}{r_{\alpha \beta}}, & \text { if } \alpha \beta \in E_{\boldsymbol{x}} \\ \mathbf{0}, & \text { otherwise. }\end{cases}
$$

\footnotetext{
${ }^{8}$ Such maps are used in rigidity theory which studies the rigidity of structures formed as an ensemble of rigid elements. See Connelly and Whiteley (1996) for an introduction to rigidity theory.

${ }^{9}$ The image $\operatorname{Im} \boldsymbol{R}$ of a map $\boldsymbol{R}: \mathcal{N}_{\boldsymbol{x}} \rightarrow \mathbb{R}^{e}$ is given by the set $\left\{\boldsymbol{R}(\boldsymbol{y}): \boldsymbol{y} \in \mathcal{N}_{\boldsymbol{x}}\right\}$.

${ }^{10}$ See Admal (2010) for a proof of uniqueness.

${ }^{11}$ Note that in the absence of a finite range of interaction, it may not be possible to represent $\widehat{\mathcal{V}}$ as a new function on $\mathcal{S}_{\boldsymbol{x}}$.
} 
We refer to $\mathcal{V}$ in Eqn. (3.8) as a potential energy extension. Note that the derivatives of $\mathcal{V}$ in Eqn. (3.9) with respect to $r_{\alpha \beta}$ for each $\alpha \beta \in E_{\boldsymbol{x}}$ are well-defined because $\mathcal{V}$ is defined in a neighborhood of $\boldsymbol{R}(\boldsymbol{y})$. This is not true of $\breve{\mathcal{V}}$ since $\partial \breve{\mathcal{V}} / \partial r_{\alpha \beta}$ is undefined. ${ }^{12}$

It is easy to verify that Eqn. (3.9) satisfies Eqns. (2.9), (3.1) and (3.2), thus qualifying to be a force decomposition that satisfies the weak and strong law of action-reaction. Moreover, the stress tensor obtained using Eqns. (3.9) and (2.13) is always symmetric. However, it is clear from the above definition that $f_{\alpha \beta}$ is not unique as it depends on the potential energy extension. (Note that different extensions of the potential energy result in the same forces $f_{\alpha}$, but different force decompositions.) In practice, the choice of the potential energy extension is made by the choice of the empirical interatomic potential, which is normally already represented as an extension. For example, pair potentials, embedded atom method potentials, three-body potentials, and so on, are potential energy extensions. On the other hand, we could in principle, take any extension and modify it, thus changing the force decomposition. Consider for example a pair potential extension with a range of interaction defined by $r_{\text {cut }}$ at a configuration $\boldsymbol{x} \in \mathbb{R}^{3 N}$,

$$
\mathcal{V}_{2}(\boldsymbol{\zeta})=\sum_{\alpha \beta \in E_{\boldsymbol{x}}} \phi\left(\zeta_{\alpha \beta}\right)
$$

where $\phi$ is a pair potential function which satisfies $\phi(r)=0$ for $r \geq r_{\text {cut }}$, and the set of arguments $\zeta=\left\{\zeta_{\alpha \beta}\right\} \in \mathbb{R}^{e}$ are arbitrary numbers corresponding to the bonds in $E_{\boldsymbol{x}}$. The value of $\mathcal{V}_{2}$ only corresponds to a physical prediction when $\zeta_{\alpha \beta}=r_{\alpha \beta}=\left\|\boldsymbol{x}_{\alpha}-\boldsymbol{x}_{\beta}\right\|$. To create an alternate extension with the same cutoff radius, we can add to $\mathcal{V}_{2}$ a function of distance arguments which is identically zero for all sets of arguments that correspond to actual physical local distances between particles. This is satisfied by the so-called Cayley-Menger (CM) determinant (Admal and Tadmor, 2010). The CM determinant, $\chi_{k}\left(r_{12}, \ldots, r_{(k-1) k}\right)$ of a set of $k$ particles represents the volume of a simplex formed by $k$ particles in a $(k-1)$-dimensional space. For example, $\chi_{5}$ represents the volume of a 5-simplex in four dimensions. If the five particles are embedded in 3-dimensions, then this volume is zero by construction. ${ }^{13}$

Returning to Eqn. (3.10), we construct the following alternate potential. Without loss of generality, we assume that in the present configuration $\boldsymbol{x}$, particles $1, \ldots, 5$ form a local cluster of size less than $r_{\text {cut }}$, or in other words all the edges connecting particles $1, \ldots, 5$ are in $E_{\boldsymbol{x}}$. We

\footnotetext{
${ }^{12}$ See Section II.B of Admal and Tadmor (2011) for a discussion of extensions of potential energy functions and an explanation of why $\partial \breve{\mathcal{V}} / \partial r_{\alpha \beta}$ is undefined.

${ }^{13}$ As a simple example, consider 3 particles constrained to move on a line. Assume the particles interact via a pair potential, $\mathcal{V}_{2}=\phi\left(\zeta_{12}\right)+\phi\left(\zeta_{13}\right)+\phi\left(\zeta_{23}\right)$. This function gives the energy of the particles when $\left(\zeta_{12}, \zeta_{13}, \zeta_{23}\right)=$ $\left(r_{12}, r_{13}, r_{23}\right)$, where $r_{12}, r_{13}$ and $r_{23}$ are the distances between the particles. An alternate extension is constructed as

$$
\mathcal{V}_{\text {alt }}=\mathcal{V}_{2}\left(\zeta_{12}, \zeta_{13}, \zeta_{23}\right)+\chi\left(\zeta_{12}, \zeta_{13}, \zeta_{23}\right),
$$

where $\chi$ is the one-dimensional $\mathrm{CM}$ determinant for 3 particles:

$$
\chi\left(\zeta_{12}, \zeta_{13}, \zeta_{23}\right)=\left(\zeta_{13}-\zeta_{23}-\zeta_{12}\right)\left(\zeta_{12}-\zeta_{13}-\zeta_{23}\right)\left(\zeta_{23}-\zeta_{12}-\zeta_{13}\right)\left(\zeta_{12}+\zeta_{13}+\zeta_{23}\right) .
$$

It is clear that $\chi$ simply reflects the geometric constraint on the particle distances. For example if the particles are ordered $x_{1}<x_{2}<x_{3}$, then the distances must satisfy $r_{12}+r_{23}=r_{13}$, this is enforced by the first term on the right-hand side above. The other terms correspond to other orderings of the particles. Thus $\chi\left(\zeta_{12}, \zeta_{13}, \zeta_{23}\right)$ is identically zero whenever the arguments $\zeta_{\alpha \beta}$ correspond to actual distances between particles, e.g. $\chi(1,2,1)$ is zero, but $\chi(1,1,1)$ (which is not physical) is non-zero.
} 
define an alternate extension as

$$
\mathcal{V}_{\text {alt }}(\boldsymbol{\zeta})=\sum_{\alpha \beta \in E_{\boldsymbol{x}}} \phi\left(\zeta_{\alpha \beta}\right)+\chi_{5}\left(\zeta_{12}, \zeta_{13}, \ldots, \zeta_{45}\right),
$$

where $\chi_{5}$ is the CM determinant for a cluster of 5 particles. Thus, $\chi_{5 \mid \mathcal{S}_{x}}=0$, and so $\mathcal{V}_{\text {alt }}$ and $\mathcal{V}_{2}$ agree on $\mathcal{S}_{\boldsymbol{x}}$. By choosing a cluster of size less than $r_{\text {cut }}$, we ensure that the cutoff radius of $\mathcal{V}_{\text {alt }}$ remains $r_{\text {cut }}$. It can be easily shown (see Admal and Tadmor (2010)) that the force decomposition resulting from $\mathcal{V}_{\text {alt }}$ differs from that obtained from $\mathcal{V}_{2}$. This leads us to the question:

Are the potential energy extensions used in practice the appropriate ones to be used for calculating the stress tensor?

In order to explore the above question, we next consider the possible quantum mechanical origins of potential energy extensions. In practice, potential energy extensions are obtained by a priori choosing a functional form for $\mathcal{V}$. The parameters in the functional form are evaluated by fitting $\mathcal{V}$ to the Born-Oppenheimer potential energy $\widehat{\mathcal{V}}$, which is obtained as an eigenvalue in the solution to the Schrödinger wave equation under the Born-Oppenheimer approximation (Tadmor and Miller, 2011). It is clear from Eqn. (3.11) that a fitted functional form can be altered using a CM determinant without affecting the fit. To our knowledge, there is no rigorous method of choosing a single functional form for $\mathcal{V}$ from the wave function obtained by solving the Schrödinger wave equation. Therefore, it is not clear how the non-uniqueness of the potential energy extension can be avoided using the quantum mechanical model. ${ }^{14}$

In the absence of a physically-motivated unique force decomposition, we now focus our attention on characterizing the non-uniqueness of the atomistic stress tensor by decomposing it into an extension-dependent part and an extension-independent part. We will then see in Section 5 that this decomposition has an interesting analog in continuum mechanics.

\section{Decomposition of the atomistic stress into an irrotational and a solenoidal part}

In this section, we propose a decomposition for the potential part of the atomistic stress tensor $\boldsymbol{\sigma}_{\mathrm{v}}$ into an extension-independent part $\boldsymbol{\sigma}_{\mathrm{v}}^{\|}$, and an extension-dependent part $\boldsymbol{\sigma}_{\mathrm{v}}^{\perp}$ for a given configuration. In other words, altering the potential energy extension does not affect $\sigma_{\mathrm{v}}^{\|}$. For the sake of simplicity we assume no periodic boundary conditions. See Appendix A, for definitions and results under periodic boundary conditions.

\subsection{Theoretical derivation of the atomistic stress decomposition}

Let $\boldsymbol{x} \in \mathbb{R}^{3 N}$ denote the configuration of interest, and let $\boldsymbol{p}=\boldsymbol{R}(\boldsymbol{x}) \in \mathcal{S}_{\boldsymbol{x}}$ denote the point on the local shape space corresponding to $\boldsymbol{x}$. This is depicted schematically in Fig. 1 with $\mathcal{S}_{\boldsymbol{x}}$ shown as a surface in $\mathbb{R}^{e}$, where $e$ is the cardinality of $E_{\boldsymbol{x}}$. Recall that the atomistic stress tensor

\footnotetext{
${ }^{14}$ Recently in his book Murdoch (2012) has addressed some of the issues we have raised in Admal and Tadmor (2010) regarding the force decomposition and its relationship to quantum mechanics. According to Murdoch, $\boldsymbol{f}_{\alpha \beta}$ is the net force on the subatomic particles of atom $\alpha$ due to the subatomic particles of atom $\beta$. Although this viewpoint treats the subatomic particles as classical particles, Murdoch argues that this approach can be extended to a quantum mechanical system. We remain skeptical of this interpretation since the only forces that can be obtained from a quantum mechanical model are the net forces on the particles $\alpha$ and $\beta$, under the Born-Oppenheimer approximation using the Hellman-Feynman theorem (Tadmor and Miller, 2011).
} 


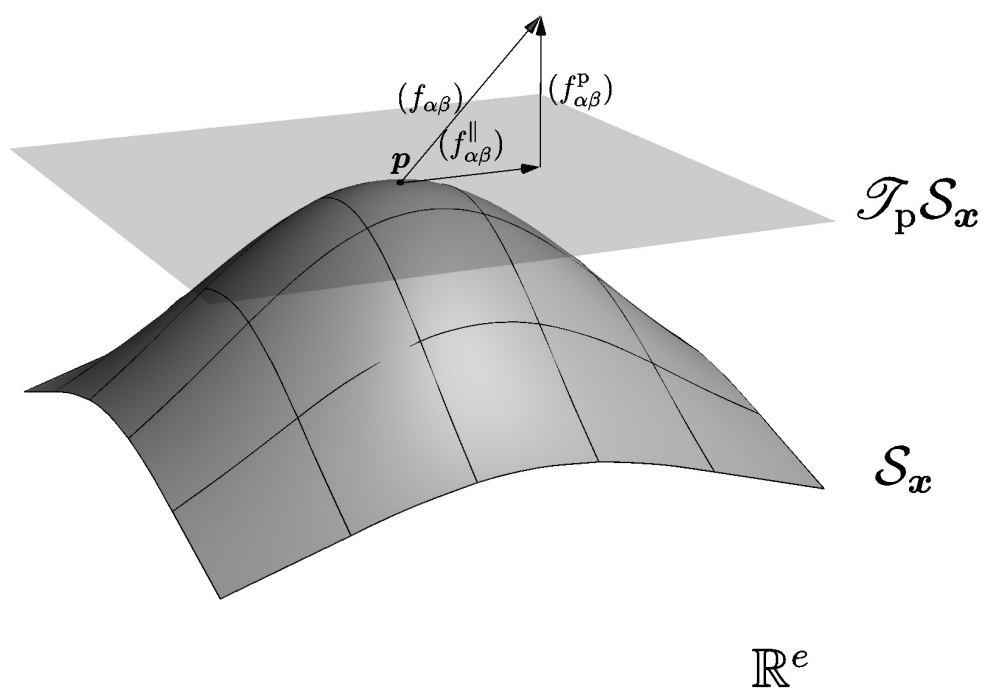

Figure 1: Schematic representation of the local shape space at a configuration $\boldsymbol{x} \in \mathbb{R}^{3 N}$ as a surface embedded in $\mathbb{R}^{e}$. In addition, the tangent space $\mathscr{T}_{\boldsymbol{p}} \mathcal{S}_{\boldsymbol{x}}$ of the local shape space at the point $\boldsymbol{p}=\boldsymbol{R}(\boldsymbol{x})$ is shown. The vectors $\left(f_{\alpha \beta}^{\|}\right) \in \mathscr{T}_{\boldsymbol{p}} \mathcal{S}_{\boldsymbol{x}}$ and $\left(f_{\alpha \beta}^{\perp}\right) \in \mathscr{T}_{\boldsymbol{p}} \mathcal{S}_{\boldsymbol{x}}^{\perp}$.

depends on the choice of the force decomposition which depends on the choice of potential energy extension. For a given extension $\mathcal{V}$, a candidate for the central force decomposition of the forces $\boldsymbol{f}_{\alpha}(\alpha=1, \ldots, N)$ is given in Eqn. (3.9), where the magnitude of force $\boldsymbol{f}_{\alpha \beta}$ is given by

$$
f_{\alpha \beta}= \begin{cases}-\left.\frac{\partial \mathcal{V}}{\partial r_{\alpha \beta}}\right|_{\boldsymbol{R}(\boldsymbol{x})} & \text { if } \alpha \beta \in E_{\boldsymbol{x}} \\ 0 & \text { otherwise }\end{cases}
$$

Let $^{15}\left(f_{\alpha \beta}\right)$ denote the ordered $e$-tuple given by the set $\left\{f_{\alpha \beta}: \alpha \beta \in E_{\boldsymbol{x}}\right\}$ with the ordering given in Eqn. (3.3). The vector $\left(f_{\alpha \beta}\right) \in \mathbb{R}^{e}$ is shown schematically in Fig. 1. Let $\mathscr{T}_{\boldsymbol{p}} \mathcal{S}_{\boldsymbol{x}}$ denote the "tangent space" to the surface $\mathcal{S}_{\boldsymbol{x}}$ at the point $\boldsymbol{p}$, as shown in Fig. $1{ }^{16}$ In order to arrive at a decomposition for the atomistic stress tensor, we split the vector $\left(f_{\alpha \beta}\right)$ by projecting it onto the

\footnotetext{
${ }^{15}$ We will use the shorthand notation $\left(\square_{\alpha \beta}\right)$ for the ordered tuple given by the set $\left\{\square_{\alpha \beta}: \alpha \beta \in E_{\boldsymbol{x}}\right\}$ with the ordering given by Eqn. (3.3).

${ }^{16}$ In mathematics, a tangent space is normally defined for a manifold (see for example Lee (2012) for definitions of manifolds and tangent spaces). Note that the surface $\mathcal{S}_{\boldsymbol{x}}$ is not a manifold due to the presence of singular configurations, such as all particles lying on a plane or a line. Thus, $\mathscr{T}_{\boldsymbol{p}} \mathcal{S}_{\boldsymbol{x}}$ is not strictly a tangent space. Nevertheless, we use this terminology, because the definition we adopt (given below in Eqn. (4.6)) is equivalent to the conventional definition at non-singular points.
} 
tangent space, and its orthogonal complement ${ }^{17} \mathscr{T}_{\boldsymbol{p}} \mathcal{S}_{\boldsymbol{x}}^{\perp}$ in $\mathbb{R}^{e}$, as shown in Fig. 1 . In other words,

$$
\left(f_{\alpha \beta}\right)=\left(f_{\alpha \beta}^{\|}\right)+\left(f_{\alpha \beta}^{\perp}\right),
$$

where $\left(f_{\alpha \beta}^{\|}\right) \in \mathscr{T}_{\boldsymbol{p}} \mathcal{S}_{\boldsymbol{x}}$, and $\left(f_{\alpha \beta}^{\perp}\right) \in \mathscr{T}_{\boldsymbol{p}} \mathcal{S}_{\boldsymbol{x}}^{\perp}$. We can now define sets of interatomic forces. For each $\alpha \beta \in E_{\boldsymbol{x}}$,

$$
\boldsymbol{f}_{\alpha \beta}=f_{\alpha \beta} \frac{\boldsymbol{x}_{\alpha}-\boldsymbol{x}_{\beta}}{r_{\alpha \beta}}, \quad \boldsymbol{f}_{\alpha \beta}^{\|}=f_{\alpha \beta}^{\|} \frac{\boldsymbol{x}_{\alpha}-\boldsymbol{x}_{\beta}}{r_{\alpha \beta}}, \quad \boldsymbol{f}_{\alpha \beta}^{\perp}=f_{\alpha \beta}^{\perp} \frac{\boldsymbol{x}_{\alpha}-\boldsymbol{x}_{\beta}}{r_{\alpha \beta}},
$$

and $\boldsymbol{f}_{\alpha \beta}=\boldsymbol{f}_{\alpha \beta}^{\|}=\boldsymbol{f}_{\alpha \beta}^{\perp}=\mathbf{0}$ for $\alpha \beta \notin E_{\boldsymbol{x}}$. From Eqns. (4.2) and (4.3), the vector $\boldsymbol{f}_{\alpha \beta}$ splits as

$$
\boldsymbol{f}_{\alpha \beta}=\boldsymbol{f}_{\alpha \beta}^{\|}+\boldsymbol{f}_{\alpha \beta}^{\perp} \text {. }
$$

Since the atomistic stress tensor given in Eqn. (2.14) depends linearly on $\boldsymbol{f}_{\alpha \beta}$, Eqn. (4.4) results in the following decomposition of the atomistic stress tensor:

$$
\sigma_{\mathrm{v}}=\sigma_{\mathrm{v}}^{\|}+\sigma_{\mathrm{v}}^{\perp}
$$

In order to formalize the geometric idea described above, we rigorously define the vector spaces $\mathscr{T}_{\boldsymbol{p}} \mathcal{S}_{\boldsymbol{x}}$ and $\mathscr{T}_{\boldsymbol{p}} \mathcal{S}_{\boldsymbol{x}}^{\perp}$ using the local rigidity map defined in Eqn. (3.5). For each point $\boldsymbol{y} \in \mathcal{N}_{\boldsymbol{x}}$, there exists a tangent map $T_{\boldsymbol{x}} \boldsymbol{R}: \mathbb{R}^{3 N} \mapsto \mathbb{R}^{e}$, which maps the tangent space of $\mathcal{N}_{\boldsymbol{x}}$ at $\boldsymbol{y}$ to the tangent space of $\mathbb{R}^{e}$ at $\boldsymbol{R}(\boldsymbol{x}) \in \mathcal{S}_{\boldsymbol{x}} \cdot{ }^{18}$ We define the vector space $\mathscr{T}_{\boldsymbol{p}} \mathcal{S}_{\boldsymbol{x}}$ as the image of the map $T_{\boldsymbol{x}} \boldsymbol{R}$ :

$$
\mathscr{T}_{\boldsymbol{p}} \mathcal{S}_{\boldsymbol{x}}:=\operatorname{Im} T_{\boldsymbol{x}} \boldsymbol{R} \subset \mathbb{R}^{e}
$$

Intuitively, the tangent map at $\boldsymbol{x}$ is a linear map that maps a "perturbation" of $\boldsymbol{x}$ in $\mathcal{N}_{\boldsymbol{x}}$ to a perturbation of $\boldsymbol{R}(\boldsymbol{x})$ in $\mathbb{R}^{e}$. A perturbation in $\boldsymbol{x}$ can be described as a vector in $\mathbb{R}^{3 N}$, while a perturbation in $\boldsymbol{R}(\boldsymbol{x})$ is described as a vector in $\mathscr{T}_{\boldsymbol{p}} \mathcal{S}_{\boldsymbol{x}}$.

We now give an explicit construction of $\mathscr{T}_{p} \mathcal{S}_{x}$ defined in Eqn. (4.6). A coordinate representation of $T_{\boldsymbol{x}} \boldsymbol{R}$, in the Cartesian coordinate system of $\mathbb{R}^{3 N}$ and $\mathbb{R}^{e}$ is given by the Jacobian matrix $\mathfrak{R}_{\boldsymbol{x}}$ evaluated at the point $\boldsymbol{x}$ for the map $\boldsymbol{R} . \mathfrak{R}_{\boldsymbol{x}}$ is a matrix of size $e \times 3 N$, with the following form. For each bond $i \in\{1,2, \ldots, e\}$, which refers to a bond, say $\alpha \beta$,

$$
\left(\mathfrak{R}_{\boldsymbol{x}}\right)_{i j}= \begin{cases}\left(\boldsymbol{x}_{\alpha}-\boldsymbol{x}_{\beta}\right)_{k} / r_{\alpha \beta}, & j \in\{3(\alpha-1)+k: k=1,2,3\} \\ \left(\boldsymbol{x}_{\beta}-\boldsymbol{x}_{\alpha}\right)_{k} / r_{\alpha \beta}, & j \in\{3(\beta-1)+k: k=1,2,3\} \\ 0, & \text { otherwise }\end{cases}
$$

From the definition of the Jacobian in Eqn. (4.7), the action of $\mathfrak{R}_{\boldsymbol{x}}$ on $\boldsymbol{u}=\left(\boldsymbol{u}_{1}, \ldots, \boldsymbol{u}_{N}\right) \in \mathbb{R}^{3 N}$

\footnotetext{
${ }^{17}$ An orthogonal complement $V^{\perp}$ of a vector subspace $V$ of $\mathbb{R}^{e}$ is defined as $V^{\perp}:=\left\{\boldsymbol{w} \in \mathbb{R}^{e}: \boldsymbol{w} \cdot \boldsymbol{v}=0\right.$ for all $\left.\boldsymbol{v} \in V\right\}$.

${ }^{18}$ Since $\mathcal{N}_{\boldsymbol{x}}$ is an open subset of $\mathbb{R}^{3 N}$, the tangent space of $\mathcal{N}_{\boldsymbol{x}}$ at any point $\boldsymbol{y} \in \mathcal{N}_{\boldsymbol{x}}$ is isomorphic to $\mathbb{R}^{3 N}$.
} 
is given by

$$
\left(\mathfrak{R}_{\boldsymbol{x}} \boldsymbol{u}\right)_{\alpha \beta}=\frac{\left(\boldsymbol{u}_{\alpha}-\boldsymbol{u}_{\beta}\right) \cdot\left(\boldsymbol{x}_{\alpha}-\boldsymbol{x}_{\beta}\right)}{r_{\alpha \beta}},
$$

where $\boldsymbol{u}$ can be viewed as a first-order approximation to a perturbation of $\boldsymbol{x}$, and $\mathfrak{R}_{\boldsymbol{x}} \boldsymbol{u}$ can be viewed as a first-order approximation to the corresponding perturbation of the interatomic distances. Since $\mathfrak{R}_{x}$ is a representation of $T_{\boldsymbol{x}} \boldsymbol{R}$, from Eqn. (4.6) we have the following explicit construction of $\mathscr{T}_{p} \mathcal{S}_{x}$ :

$$
\begin{aligned}
\mathscr{T}_{\boldsymbol{p}} \mathcal{S}_{\boldsymbol{x}} & =\operatorname{Im} \mathfrak{R}_{\boldsymbol{x}}, \\
& =\operatorname{span}\left\{\mathfrak{R}_{\boldsymbol{x}} \mid\left\{\boldsymbol{u}_{i}: i=1, \ldots, N\right\} \text { form a basis of } \mathbb{R}^{3 N}\right\} .
\end{aligned}
$$

Note that the vectors $T_{\boldsymbol{x}} \boldsymbol{R}\left(\boldsymbol{u}_{i}\right)$ in Eqn. (4.10) do not form a basis for $\mathscr{T}_{p} \mathcal{S}_{\boldsymbol{x}}$ because the Jacobian maps the perturbation vectors $\boldsymbol{u}$ corresponding to translations and rotations to the zero vector. ${ }^{19}$ Since $\left(\operatorname{Im} \Re_{\boldsymbol{x}}\right)^{\perp}=\operatorname{ker} \mathfrak{R}_{\boldsymbol{x}}^{\mathrm{T}}$, from Eqn. (4.9) we have

$$
\mathscr{T}_{p} \mathcal{S}_{x}^{\perp}=\operatorname{ker} \mathfrak{R}_{x}^{\mathrm{T}},
$$

where

$$
\operatorname{ker} \mathfrak{R}_{\boldsymbol{x}}^{\mathrm{T}}:=\left\{\boldsymbol{v} \in \mathbb{R}^{e} \mid \mathfrak{R}_{\boldsymbol{x}}^{\mathrm{T}} \boldsymbol{v}=\mathbf{0}\right\} .
$$

We now have the following characterization of $\mathscr{T}_{p} \mathcal{S}_{x}^{\perp}$. From the definition of $\mathfrak{R}_{x}$ given in Eqn. (4.7), the action of $\mathfrak{R}_{x}^{\mathrm{T}}$ on an arbitrary $\left(v_{\alpha \beta}\right) \in \mathbb{R}^{e}$, is given by ${ }^{20}$

$$
\mathfrak{R}_{\boldsymbol{x}}^{\mathrm{T}} \boldsymbol{v}=\left(\sum_{\substack{\beta \\ \alpha \beta \in E_{\boldsymbol{x}}}} v_{\alpha \beta} \frac{\boldsymbol{x}_{\alpha}-\boldsymbol{x}_{\beta}}{r_{\alpha \beta}}: \alpha=1, \ldots, N\right) .
$$

From Eqns. (4.11), (4.12) and (4.13), it is clear that $\mathscr{T}_{p} \mathcal{S}_{x}^{\perp}$ consists of all $\left(g_{\alpha \beta}\right)$ which result in zero force on each particle. In other words

$$
\left(g_{\alpha \beta}\right) \in \operatorname{ker} \mathfrak{R}_{\boldsymbol{x}}^{\mathrm{T}} \Longleftrightarrow \sum_{\substack{\beta \\ \alpha \beta \in E_{\boldsymbol{x}}}} \boldsymbol{g}_{\alpha \beta}=\mathbf{0} \quad(\alpha=1, \ldots, N) .
$$

We collect the above results in the following theorem.

Theorem 1. Let $\boldsymbol{R}: \mathcal{N}_{\boldsymbol{x}} \rightarrow \mathcal{S}_{\boldsymbol{x}}$ be a mapping of the local configuration space onto the local shape space at $\boldsymbol{x}$. Let $\left(\boldsymbol{u}_{i}: i=1, \ldots, N\right)$ be an ordered basis of $\mathbb{R}^{3 N}$. For any $\boldsymbol{x} \in \mathbb{R}^{3 N}$, the vector space $\mathscr{T}_{p} \mathcal{S}_{x}$, and its complimentary space $\mathscr{T}_{p} \mathcal{S}_{x}^{\perp}$ are described by the Jacobian $\mathfrak{R}_{x}$ of

\footnotetext{
${ }^{19}$ A perturbation $\boldsymbol{u}$ of the form $(\boldsymbol{c}, \ldots, \boldsymbol{c})$, where $\boldsymbol{c}$ is an arbitrary vector in $\mathbb{R}^{3}$ corresponds to translations, and a perturbation vector of the form $\boldsymbol{u}=\left(\boldsymbol{u}_{1}, \ldots, \boldsymbol{u}_{N}\right)$, where $\boldsymbol{u}_{\alpha}=\boldsymbol{W} \boldsymbol{x}_{\alpha}$, and $W$ is an arbitrary skew symmetric tensor corresponds to an infinitesimal rotation.

${ }^{20} \boldsymbol{v}$ can be viewed as a first-order approximation to a perturbation of $\left(f_{\alpha \beta}\right) \in \mathbb{R}^{e}$, and $\Re_{\boldsymbol{x}}^{\mathrm{T}} \boldsymbol{v}$ can be viewed as a first-order approximation to the corresponding perturbation in the net forces on the particles.
} 
the rigidity map $\boldsymbol{R}$ as

$$
\begin{aligned}
& \mathscr{T}_{\boldsymbol{p}} \mathcal{S}_{\boldsymbol{x}}=\operatorname{span}\left\{\mathfrak{R}_{\boldsymbol{x}} \boldsymbol{u}_{i}: i=1, \ldots, N\right\} \\
& \mathscr{T}_{\boldsymbol{p}} \mathcal{S}_{\boldsymbol{x}}^{\perp}=\left\{\left(g_{\alpha \beta}\right) \in \mathbb{R}^{e}: \sum_{\substack{\beta \\
\alpha \beta \in E_{\boldsymbol{x}}}} \boldsymbol{g}_{\alpha \beta}=\mathbf{0} \text { for each } \alpha=1, \ldots, N\right\} .
\end{aligned}
$$

Moreover, the vector space $\mathbb{R}^{e}$ splits as

$$
\mathbb{R}^{e}=\operatorname{Im} \Re_{\boldsymbol{x}} \oplus \operatorname{ker} \Re_{\boldsymbol{x}}^{\mathrm{T}}
$$

where $\oplus$ denotes direct sum between vector spaces.

In the following corollary, we deduce some properties of $\left(f_{\alpha \beta}^{\|}\right) \in \mathscr{T}_{p} \mathcal{S}_{x}$ and $\left(f_{\alpha \beta}^{\perp}\right) \in \mathscr{T}_{p} \mathcal{S}_{\boldsymbol{x}}^{\perp}$ and the associated $\mathbb{R}^{3 e}$-dimensional vectors $\left(\boldsymbol{f}_{\alpha \beta}^{\|}\right)$and $\left(\boldsymbol{f}_{\alpha \beta}^{\perp}\right)$, defined in Eqn. (4.3), where we use the notation in footnote 15 .

Corollary 1. For a given potential energy extension, there exist unique vectors $\left(f_{\alpha \beta}^{\|}\right) \in \mathscr{T}_{p} \mathcal{S}_{x}$, and $\left(f_{\alpha \beta}^{\perp}\right) \in \mathscr{T}_{p} \mathcal{S}_{x}^{\perp}$, such that the $\mathbb{R}^{3 e}$-dimensional vectors formed from them satisfy,

$$
\left(\boldsymbol{f}_{\alpha \beta}\right)=\left(\boldsymbol{f}_{\alpha \beta}^{\|}\right)+\left(\boldsymbol{f}_{\alpha \beta}^{\perp}\right) .
$$

Moreover, $\left(\boldsymbol{f}_{\alpha \beta}^{\|}\right)$decomposes the forces $\boldsymbol{f}_{\alpha}(\alpha=1, \ldots, N)$, and $\left(\boldsymbol{f}_{\alpha \beta}^{\perp}\right)$ decomposes a null force on each particle, i.e.

$$
\begin{gathered}
f_{\alpha}=\sum_{\substack{\beta \\
\beta \neq \alpha}} f_{\alpha \beta}^{\|}, \\
\mathbf{0}=\sum_{\substack{\beta \\
\beta \neq \alpha}} f_{\alpha \beta}^{\perp} .
\end{gathered}
$$

Additionally, $\left(\boldsymbol{f}_{\alpha \beta}^{\|}\right)$is independent of the choice of the potential extension.

Proof. Equation (4.18) follows from Eqn. (4.17) and definitions given in Eqn. (4.3). From the definition of $\boldsymbol{f}_{\alpha \beta}$ in Eqn. (3.9), we know that

$$
f_{\alpha}=\sum_{\substack{\beta \\ \beta \neq \alpha}} f_{\alpha \beta} .
$$

From Eqn. (4.18), since each $\boldsymbol{f}_{\alpha \beta}$ is decomposed as $\boldsymbol{f}_{\alpha \beta}^{\|}+\boldsymbol{f}_{\alpha \beta}^{\perp}$, we obtain

$$
f_{\alpha}=\sum_{\substack{\beta \\ \beta \neq \alpha}}\left(f_{\alpha \beta}^{\|}+f_{\alpha \beta}^{\perp}\right) .
$$


Since $\left(f_{\alpha \beta}^{\perp}\right) \in \mathscr{T}_{p} \mathcal{S}_{x}^{\perp}$, from Eqn. (4.16) it follows that $\sum_{\substack{\beta \neq \alpha \\ \beta}} \boldsymbol{f}_{\alpha \beta}^{\perp}=\mathbf{0}$. Therefore,

$$
\boldsymbol{f}_{\alpha}=\sum_{\substack{\beta \\ \beta \neq \alpha}} \boldsymbol{f}_{\alpha \beta}^{\|} .
$$

Finally, we show that $\left(f_{\alpha \beta}^{\|}\right)$is independent of the choice of extension. Suppose that we have an alternate extension, that results in

$$
\boldsymbol{f}_{\alpha}=\sum_{\substack{\beta \\ \beta \neq \alpha}} \tilde{\boldsymbol{f}}_{\alpha \beta}^{\|},
$$

which is an analog of Eqn. (4.21) for the alternate extension. Subtracting Eqn. (4.22) from Eqn. (4.21), we obtain

$$
\mathbf{0}=\sum_{\substack{\beta \\ \beta \neq \alpha}} \boldsymbol{f}_{\alpha \beta}^{\|}-\tilde{f}_{\alpha \beta}^{\|}
$$

Since $\left(f_{\alpha \beta}^{\|}\right)$and $\left(\tilde{f}_{\alpha \beta}^{\|}\right)$belong to $\operatorname{Im} \Re_{\boldsymbol{x}}$, it follows that $\left(f_{\alpha \beta}^{\|}-\tilde{f}_{\alpha \beta}^{\|}\right) \in \operatorname{Im} \Re_{\boldsymbol{x}}$. On the other hand, from Eqns. (4.16) and (4.23), we conclude that $\left(f_{\alpha \beta}^{\|}-\tilde{f}_{\alpha \beta}^{\|}\right) \in \operatorname{ker} \mathfrak{R}_{x}^{\mathrm{T}}$. Since $\operatorname{Im} \mathfrak{R} \cap \operatorname{ker} \mathfrak{R}_{x}^{\mathrm{T}}=$ $\{\mathbf{0}\}$, it follows that for each bond, $f_{\alpha \beta}^{\|}-\tilde{f}_{\alpha \beta}^{\|}=0$, which is the desired result.

We now define $\sigma_{\mathrm{v}}^{\|}$and $\sigma_{\mathrm{v}}^{\perp}$ as the distributions obtained by substituting in $\boldsymbol{f}_{\alpha \beta}^{\|}$and $\boldsymbol{f}_{\alpha \beta}^{\perp}$, respectively, in place of $f_{\alpha \beta}$ in the right-hand side of Eqn. (2.17). Since $\sigma_{\mathrm{v}}$ defined in Eqn. (2.17) depends linearly on the forces $f_{\alpha \beta}$, it follows that

$$
\sigma_{\mathrm{v}}=\sigma_{\mathrm{v}}^{\|}+\sigma_{\mathrm{v}}^{\perp} .
$$

We define the stress tensor fields corresponding to the distributions $\sigma_{\mathrm{v}}^{\|}$and $\sigma_{\mathrm{v}}^{\perp}$ as

$$
\begin{aligned}
& \boldsymbol{\sigma}_{w, \mathrm{v}}^{\|}:=w \star \boldsymbol{\sigma}_{\mathrm{v}}^{\|}, \\
& \boldsymbol{\sigma}_{w, \mathrm{v}}^{\perp}:=w \star \boldsymbol{\sigma}_{\mathrm{v}}^{\perp} .
\end{aligned}
$$

In the following two corollaries, we obtain properties of $\boldsymbol{\sigma}_{w, \mathrm{v}}^{\|}$and $\boldsymbol{\sigma}_{w, \mathrm{v}}^{\perp}$.

Corollary 2. The tensors $\boldsymbol{\sigma}_{w, \mathrm{v}}, \boldsymbol{\sigma}_{w, \mathrm{v}}^{\|}$and $\boldsymbol{\sigma}_{w, \mathrm{v}}^{\perp}$, satisfy the equations

$$
\begin{aligned}
& \operatorname{div}_{\boldsymbol{x}} \boldsymbol{\sigma}_{w, \mathrm{v}}(\boldsymbol{x}, t)=\operatorname{div}_{\boldsymbol{x}} \boldsymbol{\sigma}_{w, \mathrm{v}}^{\|}(\boldsymbol{x}, t)=\sum_{\alpha} \boldsymbol{f}_{\alpha} w\left(\boldsymbol{x}_{\alpha}(t)-\boldsymbol{x}\right), \\
& \operatorname{div}_{\boldsymbol{x}} \boldsymbol{\sigma}_{w, \mathrm{v}}^{\perp}(\boldsymbol{x}, t)=\mathbf{0} .
\end{aligned}
$$

Moreover, $\sigma_{w, \mathrm{v}}^{\|}$is independent of the potential energy extension.

Proof. From Eqn. (4.25) we see that expressions $\sigma_{w, \mathrm{v}}^{\|}$and $\sigma_{w, \mathrm{v}}^{\perp}$ are obtained from Eqn. (2.13) by replacing the forces $\boldsymbol{f}_{\alpha \beta}$ with $\boldsymbol{f}_{\alpha \beta}^{\|}$and $\boldsymbol{f}_{\alpha \beta}^{\perp}$, respectively. Therefore, from Corollary $1, \sigma_{w, \mathrm{v}}^{\|}$ 
is independent of the potential energy extension. Moreover, since $\sigma_{w, \mathrm{v}}$ satisfies Eqn. (2.10), we have

$$
\begin{aligned}
\operatorname{div}_{\boldsymbol{x}} \boldsymbol{\sigma}_{w, \mathrm{v}}^{\|}(\boldsymbol{x}, t) & =\sum_{\substack{\alpha, \beta \\
\alpha<\beta}} \boldsymbol{f}_{\alpha \beta}^{\|} w\left(\boldsymbol{x}_{\alpha}-\boldsymbol{x}\right) \\
\operatorname{div}_{\boldsymbol{x}} \boldsymbol{\sigma}_{w, \mathrm{v}}^{\perp}(\boldsymbol{x}, t) & =\sum_{\substack{\alpha, \beta \\
\alpha<\beta}} \boldsymbol{f}_{\alpha \beta}^{\perp} w\left(\boldsymbol{x}_{\alpha}-\boldsymbol{x}\right) .
\end{aligned}
$$

Using Eqns. (4.19) and (4.20), equation Eqn. (4.26) follows from the above equalities.

Corollary 3. Let $\boldsymbol{x} \in \mathbb{R}^{3 N}$ be a configuration for which $\boldsymbol{f}_{\alpha}=\mathbf{0}$ for each particle $\alpha$. For such a configuration, $\boldsymbol{\sigma}_{\mathrm{v}}$ has the following trivial decomposition: $\boldsymbol{\sigma}_{\mathrm{v}}^{\perp}=\boldsymbol{\sigma}_{\mathrm{v}}$ and $\boldsymbol{\sigma}_{\mathrm{v}}^{\|}=\mathbf{0}$.

Proof. Since the net force on each particle is zero, we have $\sum_{\substack{\beta \neq \alpha \\ \beta \neq \alpha}} \boldsymbol{f}_{\alpha \beta}=\mathbf{0}$. Therefore, it follows from Eqn. (4.16) that $\left(f_{\alpha \beta}\right) \in \mathscr{T}_{p} \mathcal{S}_{x}^{\perp}$. Moreover, from Eqn. (4.17) in Theorem 1, we have $\left(f_{\alpha \beta}^{\|}\right)=\left(f_{\alpha \beta}\right)$ and $\left(f_{\alpha \beta}^{\perp}\right)=\mathbf{0}$. Therefore, $\boldsymbol{\sigma}_{w, \mathrm{v}}^{\|}=\mathbf{0}$ and $\boldsymbol{\sigma}_{w, \mathrm{v}}^{\perp}=\boldsymbol{\sigma}_{w, \mathrm{v}}$.

From Corollaries 2 and 3, we see that $\boldsymbol{\sigma}_{w, \mathrm{v}}^{\perp}$ is always divergence-free and $\boldsymbol{\sigma}_{w, \mathrm{v}}^{\|}$is zero in the absence of external loading. Therefore, we refer to $\sigma_{w, \mathrm{v}}^{\perp}$ as the solenoidal part of the atomistic stress and $\boldsymbol{\sigma}_{w, \mathrm{v}}^{\|}$as the irrotational part of the atomistic stress. The solenoidal part $\boldsymbol{\sigma}_{w, \mathrm{v}}^{\perp}$ is extension-dependent and thus non-unique, whereas the irrotational part $\sigma_{w, \mathrm{v}}^{\|}$is uniquely defined. The terms irrotational and solenoidal are borrowed from the Helmholtz decomposition of vector fields. The choice of this terminology is made clear in the next section where we discuss the Beltrami representation of the continuum Cauchy stress tensor, which is a Helmholtz-like decomposition for symmetric tensor fields.

It is interesting to note from Corollary 3 that the atomistic stress in a relaxed configuration of a system with defects is extension-dependent. ${ }^{21}$ The fact that the atomistic stress obtained using interatomic potentials for self-equilibrated systems are often in agreement with continuum solutions despite being extension-dependent suggests that by construction potentials carry information on the nature of bonding in the material. In other words, in addition to reproducing the forces on atoms predicted by quantum mechanics, interatomic forces contain additional information related to electronic wavefunctions. Thus the question of choosing an appropriate extension may possibly be answered by exploring derivations of the stress for quantum systems that explicitly include electron density fields.

The decomposition derived in this section has a potential application in the systematic reduction of noise in atomistic fields. Recall from Section 2 that one of the sources of non-uniqueness in the Murdoch-Hardy procedure is the choice of the weighting function. The size of the weighting function is chosen to average out the noise which exists due to the discrete nature of the atomistic system, and at the same time preserve the macroscopic feature of the continuum field. A recent article by Ulz et al. (2013) proposes an optimal size for the support of the weighting function based on the correlation length of the potential energy function. In our numerical tests, described in Section 7, we observe an interesting feature of scale separation in the decomposition of the atomistic stress field. This suggests an alternative method to systematically reduce

\footnotetext{
${ }^{21}$ The authors would like to thank the anonymous reviewer for pointing out this interesting feature.
} 
noise which may not be accessible using a single weighting function. This is discussed further in Section 7.

\subsection{A practical algorithm of the atomistic stress decomposition}

We now describe a practical algorithm for decomposing the atomistic stress tensor into its solenoidal and irrotational parts. This requires as to decompose the forces $\left(\boldsymbol{f}_{\alpha \beta}\right)$ according to Eqn. (4.18).

The input to algorithm is the configuration of the system $\boldsymbol{x}$, the cutoff radius $r_{\text {cut }}$, the potential energy function $\mathcal{V}$, and the weighting function $w$. The cutoff radius and the configuration are used collect the edges in $E_{\boldsymbol{x}}$. Using $E_{\boldsymbol{x}}$ and $\boldsymbol{x}$, the $e \times 3 N$ sparse matrix $\mathfrak{R}_{\boldsymbol{x}}$ defined in Eqn. (4.7) is assembled in sparse format. Each component of $\left(f_{\alpha \beta}\right)$ defined in Eqn. (4.1) is calculated using the given potential energy extension and assembled as an $e \times 1$ array. The vector $\left(f_{\alpha \beta}^{\|}\right)$is obtained by projecting it onto the tangent space $\mathscr{T}_{\boldsymbol{p}} \mathcal{S}_{\boldsymbol{x}}$. The projection is implemented as a minimization problem:

$$
\left(f_{\alpha \beta}^{\|}\right)=\underset{\boldsymbol{h} \in \mathscr{T}_{p} \mathcal{S}_{\boldsymbol{x}}}{\operatorname{argmin}}\left\|\boldsymbol{h}-\left(f_{\alpha \beta}\right)\right\| .
$$

Referring to Eqn. (4.15), we see that this is equivalent to setting

$$
\left(f_{\alpha \beta}^{\|}\right)=\mathfrak{R}_{\boldsymbol{x}} u_{*},
$$

where $u_{*}$ is the solution to the following least-squares problem:

$$
\boldsymbol{u}_{*}=\underset{\boldsymbol{u} \in \mathbb{R}^{3 N}}{\operatorname{argmin}}\left\|\Re_{\boldsymbol{x}} \boldsymbol{u}-\left(f_{\alpha \beta}\right)\right\| \text {. }
$$

In order to solve Eqn. (4.31), we use a linear least squares solver based on the Golub-Kahan bidiagonalization process (Paige and Saunders, 1982). Once $\left(f_{\alpha \beta}^{\|}\right)$are computed, The orthogonal forces follow as $f_{\alpha \beta}^{\perp}=f_{\alpha \beta}-f_{\alpha \beta}^{\|}$. The vectors $\boldsymbol{f}_{\alpha \beta}, \boldsymbol{f}_{\alpha \beta}^{\|}$and $\boldsymbol{f}_{\alpha \beta}^{\perp}$ are then computed from Eqn. (4.3), and the stress fields $\boldsymbol{\sigma}_{w, \mathrm{v}}, \boldsymbol{\sigma}_{w, \mathrm{v}}^{\|}$and $\boldsymbol{\sigma}_{w, \mathrm{v}}^{\perp}$ follow using the weighting function $w$. The above procedure is summarized in Algorithm 1.

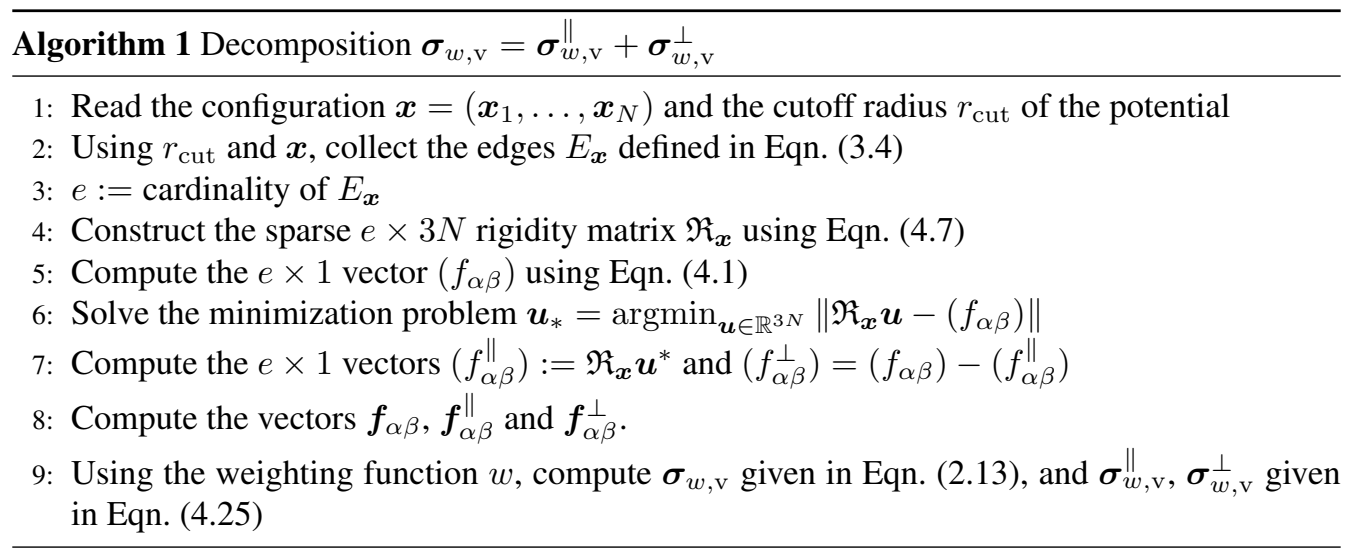


A KIM-compliant Fortran program for performing the decomposition is available as part of the supplementary content accompanying this article. ${ }^{22}$

In the next section, we discuss an analogous decomposition of the Cauchy stress tensor in continuum mechanics using the generalized Beltrami representation.

\section{A Generalized Beltrami representation for the continuum Cauchy stress tensor}

Let $\Omega \subset \mathbb{R}^{3}$ be a bounded open subset with a smooth boundary, and let $\mathscr{S}, \mathscr{V}$ and SYM denote the space of smooth scalar, vector and symmetric tensor fields on $\Omega$, respectively. It is well-known from the Helmholtz decomposition of vector fields that any $\boldsymbol{u} \in \mathscr{V}$ can be represented in terms of a scalar field $\phi \in \mathscr{S}$, and a vector field $\psi \in \mathscr{V}$ :

$$
\boldsymbol{u}(\boldsymbol{x})=\nabla \phi(\boldsymbol{x})+\operatorname{curl} \boldsymbol{\psi}(\boldsymbol{x}), \quad \boldsymbol{x} \in \Omega .
$$

For a given pointwise stable ${ }^{23}$ elasticity tensor $\mathbb{C}^{*}$, a representation similar to Eqn. (5.1) exists for a tensor field $\boldsymbol{T} \in \mathrm{SYM}$, given by

$$
\boldsymbol{T}(\boldsymbol{x})=\operatorname{curl}(\operatorname{curl} \boldsymbol{\Psi}(\boldsymbol{x}))+\frac{1}{2} \mathbb{C}^{*}\left(\nabla \boldsymbol{v}(\boldsymbol{x})+\nabla \boldsymbol{v}^{\mathrm{T}}(\boldsymbol{x})\right), \quad \boldsymbol{x} \in \Omega,
$$

where $\Psi \in \mathrm{SYM}$ and $\boldsymbol{v} \in \mathscr{V}$. The representation given in Eqn. (5.2) is commonly referred to as a "generalized Beltrami representation" (Gurtin, 1963; Fosdick and Schuler, 2003; Fosdick and Royer-Carfagni, 2005). ${ }^{24}$ Within elasticity theory, Beltrami (1892) discovered that in the absence of body force field, a continuum Cauchy stress tensor $\sigma_{\mathrm{c}}$ of the form

$$
\boldsymbol{\sigma}_{\mathrm{c}}(\boldsymbol{x})=\operatorname{curl}(\operatorname{curl} \boldsymbol{\Psi})(\boldsymbol{x}),
$$

identically satisfies the equilibrium equation, $\operatorname{div}_{\boldsymbol{x}} \sigma_{\mathrm{c}}=\mathbf{0}$. The representation given in Eqn. (5.3) is commonly referred to as a "Beltrami representation." A question then arose regarding the validity of the converse statement: Does $\operatorname{div}_{\boldsymbol{x}} \boldsymbol{T}=\mathbf{0}, \boldsymbol{T} \in \mathrm{SYM}$ imply that $\boldsymbol{T}$ has the Beltrami representation given in Eqn. (5.3)? Gurtin (1963) showed that there exist symmetric tensor fields satisfying $\operatorname{div}_{\boldsymbol{x}} \boldsymbol{T}=\mathbf{0}$, which do not have a Beltrami representation. In particular, it was shown that a symmetric tensor field $\boldsymbol{T}$ has a Beltrami representation if and only if either of the following two conditions are satisfied:

1. $\operatorname{div}_{\boldsymbol{x}} \boldsymbol{T}=\mathbf{0}$ in $\Omega$, and $\Omega$ is non-periphractic ${ }^{25}$.

\footnotetext{
${ }^{22}$ The Open Knowledgebase of Interatomic Models (KIM) (Tadmor et al., 2011, 2013) is a project focused on creating standards for atomistic simulations including an application programming interface (API) for information exchange between atomistic simulation codes and interatomic potentials. The Fortran program accompanying this paper works with all interatomic potentials stored in the OpenKIM Repository at https://openkim.org, which are compatible with the KIM API standard.

${ }^{23}$ An elasticity tensor $\mathbb{C}^{*}$ is pointwise stable if for every symmetric tensor $\epsilon, \mathbb{C}^{*} \epsilon \cdot \epsilon>0$.

${ }^{24}$ The generalized-Beltrami representation discussed in Gurtin (1963); Fosdick and Schuler (2003); Fosdick and Royer-Carfagni (2005) takes $\mathbb{C}^{*}$ to be the fourth-order identity tensor.

${ }^{25}$ A periphractic domain refers to domains which have holes, but not holes that pierce completely through it.
} 
2. For every closed surface $\mathcal{S} \subset \Omega$,

$$
\begin{aligned}
\int_{\mathcal{S}} \boldsymbol{T}(\boldsymbol{x}) \boldsymbol{n}(\boldsymbol{x}) d a & =\mathbf{0} \\
\int_{\mathcal{S}} \boldsymbol{x} \times \boldsymbol{T}(\boldsymbol{x}) \boldsymbol{n}(\boldsymbol{x}) d a & =\mathbf{0},
\end{aligned}
$$

where $\boldsymbol{n}(\boldsymbol{x})$ is a unit normal field on $\mathcal{S}$, and $\times$ denotes the vector cross product.

For an example in which conditions 1 and 2 are not satisfied, consider a periphractic domain such as a hollow sphere of positive thickness. Construct a boundary-value problem on this domain by specifying non-zero tractions on the inner and outer surfaces, such that the integrals of tractions on the inner and outer surfaces are non-zero, while the net traction on the body is zero. A linear elastic solution for the given boundary-value problem results in a stress tensor field that does not have a Beltrami representation. Since Eqn. (5.2) "completes" the Beltrami representation, it is a generalization of the Beltrami representation.

Now let us consider the uniqueness of the Helmholtz and Beltrami representations. Note that for an unbounded domain, the Helmholtz decomposition in Eqn. (5.1) applies only to smooth functions with a decay property at infinity given by

$$
\boldsymbol{u}(\boldsymbol{x})=\boldsymbol{c}+\boldsymbol{o}\left(\|\boldsymbol{x}\|^{-\delta}\right),
$$

for some $\delta>0$ and $c \in \mathbb{R}^{3}$. (See Gurtin (1962) for a proof of the Helmholtz decomposition for unbounded domains.) For a given $\boldsymbol{u}$ that decays at infinity, the fields $\nabla \phi$ and curl $\boldsymbol{\psi}$ are uniquely defined. ${ }^{26}$ This follows from the following argument. Assume that there exists an alternate decomposition of $\boldsymbol{u}$ given by $\boldsymbol{u}=\nabla \tilde{\phi}+\operatorname{curl} \tilde{\boldsymbol{\psi}}$, for some scalar and vector fields $\tilde{\phi}$ and $\tilde{\psi}$ respectively, such that $\nabla \phi \not \equiv \nabla \tilde{\phi}$. This implies $\operatorname{div} \nabla(\phi-\tilde{\phi})=0$, or in other words, $\phi-\tilde{\phi}$ is a harmonic function. Since $\phi$ and $\tilde{\phi}$ satisfy the decay property given in Eqn. (5.6), $\phi-\tilde{\phi}$ is bounded. From the maximum principle for harmonic functions (Renardy and Rogers, 2004), we know that the only harmonic function on $\mathbb{R}^{3}$ that is bounded is a constant function. Therefore, $\phi$ and $\tilde{\phi}$ differ by a constant, which implies $\nabla \phi \equiv \nabla \tilde{\phi}$, a contradiction. In contrast, the decomposition given in Eqn. (5.1) is not unique for bounded domains. This can be easily seen by altering an existing decomposition $\boldsymbol{u}=\nabla \phi+\operatorname{curl} \boldsymbol{\psi}$ to $\boldsymbol{u}=\nabla(\phi+h)+\operatorname{curl}(\boldsymbol{\psi}-\widehat{\boldsymbol{\psi}})$, where $h$ is a harmonic potential on $\Omega$, and $\widehat{\psi}$ is a vector field such that ${ }^{27} \operatorname{curl} \widehat{\psi}=\nabla h$. A unique decomposition can be obtained for bounded domains, if an additional condition

$$
\operatorname{curl} \boldsymbol{\psi}(\boldsymbol{x}) \cdot \boldsymbol{n}(\boldsymbol{x})=0, \quad \boldsymbol{x} \in \partial \Omega,
$$

is imposed on $\psi$. We therefore have the following orthogonal decomposition of $\mathscr{V}$, equipped with the $L^{2}$ inner product for bounded domains:

$$
\mathscr{V}=\{\nabla \phi: \phi \in \mathscr{S}\} \oplus^{\perp}\{\operatorname{curl} \boldsymbol{\psi}: \operatorname{curl} \boldsymbol{\psi} \cdot \boldsymbol{n}=0 \text { on } \partial \Omega, \boldsymbol{\psi} \in \mathscr{V}\} .
$$

See Ghiloni (2010) for a discussion on various other Helmholtz orthogonal decompositions of square-integrable vector fields.

\footnotetext{
${ }^{26}$ Note that this does not mean that $\phi$ and $\boldsymbol{\psi}$ are unique.

${ }^{27}$ Since $\operatorname{div}(\nabla h)=0$, there exists a vector field $\widehat{\boldsymbol{\psi}}$ such that $\operatorname{curl} \widehat{\boldsymbol{\psi}}=\nabla h$. For a proof, see Girault and Raviart (1979).
} 
Similar to the above discussion on vector fields, the decomposition for tensor fields given in Eqn. (5.2) is not unique. This can be seen from the following example. Consider a solution for the displacement field $\widehat{\boldsymbol{v}}$ for a linear elastic boundary-value problem on a non-periphractic domain with the elasticity tensor $\mathbb{C}^{*}$. Since $\mathbb{C}^{*}$ is strongly elliptic, it follows that a single class of solutions exist that are related to each other by rigid body motion. Then the resulting continuum stress tensor field for any solution $\widehat{\boldsymbol{v}}$ in this class is given by

$$
\widehat{\boldsymbol{\sigma}}=\frac{1}{2} \mathbb{C}^{*}\left(\nabla \widehat{\boldsymbol{v}}+\nabla \widehat{\boldsymbol{v}}^{\mathrm{T}}\right)
$$

On the other hand, since $\operatorname{div} \widehat{\boldsymbol{\sigma}}=\mathbf{0}$, and the domain is non-periphractic, from condition 1 we know that there exists a $\widehat{\Psi} \in \mathrm{SYM}$, such that

$$
\widehat{\boldsymbol{\sigma}}=\operatorname{curl}(\operatorname{curl} \widehat{\boldsymbol{\Psi}})
$$

Using Eqns. (5.9) and (5.10), we can now alter an existing decomposition for a tensor field $\boldsymbol{T} \in$ SYM, given in Eqn. (5.2), by changing $\boldsymbol{\Psi}$ and $\boldsymbol{v}$ to $\boldsymbol{\Psi}+\widehat{\boldsymbol{\Psi}}$ and $\boldsymbol{v}-\widehat{\boldsymbol{v}}$, respectively. A unique decomposition of a tensor field is obtained if we impose the following additional condition on $\Psi$ :

$$
\operatorname{curl}(\operatorname{curl} \boldsymbol{\Psi}(\boldsymbol{x})) \boldsymbol{n}(\boldsymbol{x})=\mathbf{0}, \quad \boldsymbol{x} \in \partial \Omega .
$$

Equation (5.11) is an analog of Eqn. (5.7) for tensor fields. Therefore, the continuum Cauchy stress can be uniquely decomposed as

$$
\sigma_{\mathrm{c}}=\sigma_{\mathrm{c}}^{\|}+\sigma_{\mathrm{c}}^{\perp}
$$

where for some $\boldsymbol{v} \in \mathscr{V}$ and $\boldsymbol{\Psi} \in \mathrm{SYM}$,

$$
\begin{aligned}
\boldsymbol{\sigma}_{\mathrm{c}}^{\|}(\boldsymbol{x}) & =\frac{1}{2} \mathbb{C}^{*}\left(\nabla \boldsymbol{v}(\boldsymbol{x})+\nabla \boldsymbol{v}^{\mathrm{T}}(\boldsymbol{x})\right), & \boldsymbol{x} \in \Omega, \\
\boldsymbol{\sigma}_{\mathrm{c}}^{\perp}(\boldsymbol{x}) & =\operatorname{curl}(\operatorname{curl} \boldsymbol{\Psi}(\boldsymbol{x})), & \boldsymbol{x} \in \Omega, \\
\left(\boldsymbol{\sigma}_{\mathrm{c}}^{\perp} \boldsymbol{n}\right)(\boldsymbol{x}) & =\mathbf{0}, & \boldsymbol{x} \in \partial \Omega .
\end{aligned}
$$

Moreover, it can be easily shown that SYM equipped with the $L_{\mathbb{C}^{*}}^{2}$ inner product given by ${ }^{28}$

$$
\langle\boldsymbol{U}, \boldsymbol{V}\rangle=\int_{\Omega}\left(\mathbb{C}^{*}\right)^{-1} \boldsymbol{U}(\boldsymbol{x}) \cdot \boldsymbol{V}(\boldsymbol{x}) d \boldsymbol{x}
$$

has the following orthogonal decomposition:

$$
\begin{aligned}
\mathrm{SYM}= & \left\{\mathbb{C}^{*}\left(\nabla \boldsymbol{v}+\nabla \boldsymbol{v}^{\mathrm{T}}\right): \boldsymbol{v} \in \mathscr{V}\right\} \oplus^{\perp} \\
& \{\operatorname{curl} \operatorname{curl} \boldsymbol{\Psi}: \operatorname{curl}(\operatorname{curl} \boldsymbol{\psi}) \boldsymbol{n}=\mathbf{0} \text { on } \partial \Omega, \boldsymbol{\Psi} \in \mathrm{SYM}\} .
\end{aligned}
$$

It is clear from Eqn. (5.13) that $\sigma_{\mathrm{c}}^{\|}$is the "irrotational" part, and $\sigma_{\mathrm{c}}^{\perp}$ is the traction-free "solenoidal part" of $\sigma_{\mathrm{c}}$. Note that the decomposition given in Eqn. (5.12) is unique for a given $\mathbb{C}^{*}$. In the next

\footnotetext{
${ }^{28}$ Since $\mathbb{C}^{*}$ is assumed to be pointwise stable, it follows that $\mathbb{C}^{*}$ is invertible.
} 
section, we show that for a specially chosen $\mathbb{C}^{*}$ that is independent of the potential energy and only depends on the geometry of the underlying crystalline structure, the resulting continuum decomposition is analogous to the atomistic decomposition discussed in Section 4.

\section{Relationship between the atomistic and continuum decompositions of the Cauchy stress tensor}

In this section, we establish the connection between the decomposition of the atomistic stress introduced in Section 4, and the decomposition of the continuum stress discussed in Section 5. Recall from Section 5 that for a given stress distribution $\sigma_{\mathrm{c}}$ on $\Omega, \sigma_{c}^{\|}$is obtained by choosing a $\mathbb{C}^{*}$, and solving the associated linear elastic problem (see Eqn. (5.13)) on $\Omega$. Similarly, recall from Section 4 that for an atomistic system $\boldsymbol{\sigma}_{w, v}^{\|}$is obtained by solving a minimization problem (see Eqn. (4.31)) which is equivalent to solving the following system of linear equations: ${ }^{29}$

$$
\mathfrak{R}_{\boldsymbol{x}}^{\mathrm{T}} \mathfrak{R}_{\boldsymbol{x}} \boldsymbol{u}=\boldsymbol{f}
$$

where $\boldsymbol{x} \in \mathbb{R}^{3 N}$ is the configuration of interest, and $\boldsymbol{f}=\left(\boldsymbol{f}_{1}, \ldots, \boldsymbol{f}_{N}\right) \in \mathbb{R}^{3 N}$. Therefore, in order to establish the analogy between $\sigma_{c}^{\|}$and $\sigma_{w, v}^{\|}$, we postulate that the choice of $\mathbb{C}^{*}$ should be based on the energy function associated with the system of equations given in Eqn. (6.1). From the expression for $\mathfrak{R}$ given in Eqn. (4.7) (or Eqn. (A.3) under periodic boundary conditions), it follows that the solution to Eqn. (6.1) is the minimizer of the following energy function:

$$
\widehat{\mathcal{E}}(\boldsymbol{u})=\mathcal{E}\left(\left(r_{\alpha \beta}\right)\right)=\frac{1}{2} \sum_{\alpha \beta \in E_{\boldsymbol{x}}}\left(r_{\alpha \beta}-\bar{r}_{\alpha \beta}\right)^{2}-\sum_{\alpha} \boldsymbol{f}_{\alpha} \cdot \boldsymbol{u}_{\alpha}
$$

where $\alpha \beta \in E_{\boldsymbol{x}}, \bar{r}_{\alpha \beta}$ and $r_{\alpha \beta}$ are norms of the vectors $\overline{\boldsymbol{r}}_{\alpha \beta}=\boldsymbol{x}_{\alpha}-\boldsymbol{x}_{\beta}$ and $\boldsymbol{r}_{\alpha \beta}=\left(\boldsymbol{x}_{\alpha}+\boldsymbol{u}_{\alpha}\right)-$ $\left(\boldsymbol{x}_{\beta}+\boldsymbol{u}_{\beta}\right)$, respectively, ${ }^{30}$ and $\mathcal{E}$ is an extension of $\widehat{\mathcal{E}}$. We note that although the first term on the right-hand-side of Eqn. (6.2) is purely geometric, it has units of energy. In other words, the "spring constant" associated with each bond is taken as unity. In order to identify $\boldsymbol{\sigma}_{\mathrm{c}}^{\|}$with $\boldsymbol{\sigma}_{w, v}^{\|}$, we choose $\mathbb{C}^{*}$ as the microscopic elasticity tensor (see Tadmor and Miller (2011) for a derivation of the microscopic elasticity tensor for an atomistic system) resulting from the energy $\mathcal{E}$. The

\footnotetext{
${ }^{29}$ The system of equations given in Eqn. (6.1) are obtained from Eqn. (4.31) by taking the derivative of $\| \Re_{\boldsymbol{x}} \boldsymbol{u}-$ $\left(f_{\alpha \beta}\right) \|$ with respect to each $\boldsymbol{u}_{\alpha}$ and noting from the action of $\mathfrak{R}_{\boldsymbol{x}}^{\mathrm{T}}$ given in Eqn. (4.13) that $\mathfrak{R}^{\mathrm{T}}\left(f_{\alpha \beta}\right)=\boldsymbol{f}$. Also, note that under periodic boundary conditions (see Appendix A) $\boldsymbol{x} \in \mathbb{R}^{3 N+3}, \mathfrak{R}_{\boldsymbol{x}}$ is a $e \times(3 N+3)$ matrix, and from the definition of $\Re_{\boldsymbol{x}}$ in Eqn. (A.3) it follows that $\boldsymbol{f}=\left(\boldsymbol{f}_{1}, \ldots, \boldsymbol{f}_{N}, f_{L_{1}}, f_{L_{2}}, f_{L_{3}}\right)$, where $f_{L_{i}}=\partial \widehat{\mathcal{V}} / \partial L_{i}(i=1,2,3)$ and $L_{i}$ are the periodic orthogonal box side lengths.

${ }^{30}$ Under periodic boundary conditions, the expression for $\Re$ is given by Eqn. (A.3), and the vectors $\boldsymbol{r}_{\alpha \beta}$ and $\overline{\boldsymbol{r}}_{\alpha \beta}$ are replaced by $\boldsymbol{r}_{\alpha \beta}^{\#}$ and $\overline{\boldsymbol{r}}_{\alpha \beta}^{\#}$, respectively. See Appendix A.
} 
microscopic elasticity tensor $\mathbb{C}$ resulting from a potential energy $\widehat{\mathcal{V}}$ at $0 \mathrm{~K}$ is given by

$$
\begin{array}{r}
\mathbb{C}_{i j k l}=\frac{1}{V}\left[\left.\sum_{\substack{\alpha, \beta \\
\alpha<\beta}} \sum_{\substack{\gamma<\delta \\
\gamma<\delta}} \frac{\partial^{2} \mathcal{V}}{\partial r_{\alpha \beta} \partial r_{\gamma \delta}}\right|_{\left(\bar{r}_{\alpha \beta)}\right.} \frac{\overline{\boldsymbol{r}}_{\alpha \beta} \otimes \overline{\boldsymbol{r}}_{\alpha \beta} \otimes \overline{\boldsymbol{r}}_{\gamma \delta} \otimes \overline{\boldsymbol{r}}_{\gamma \delta}}{\bar{r}_{\alpha \beta} \bar{r}_{\gamma \delta}}\right. \\
\left.-\left.\sum_{\substack{\alpha, \beta \\
\alpha<\beta}} \frac{\partial \mathcal{V}}{\partial r_{\alpha \beta}}\right|_{\left.\bar{r}_{\alpha \beta}\right)} \frac{\overline{\boldsymbol{r}}_{\alpha \beta} \otimes \overline{\boldsymbol{r}}_{\alpha \beta} \otimes \overline{\boldsymbol{r}}_{\alpha \beta} \otimes \overline{\boldsymbol{r}}_{\alpha \beta}}{\bar{r}_{\alpha \beta}^{3}}\right],
\end{array}
$$

where $\mathcal{V}$ is an extension of $\widehat{\mathcal{V}}$. Although the above definition is described in terms of a potential energy extension, it can be easily shown that $\mathbb{C}_{i j k l}$ defined in Eqn. (6.3) is independent of the choice of extension. We choose $\mathbb{C}^{*}$ as the microscopic elasticity tensor resulting by substituting into Eqn. (6.3), $\mathcal{E}$ from Eqn. (6.2) in place of $\mathcal{V}$. Since

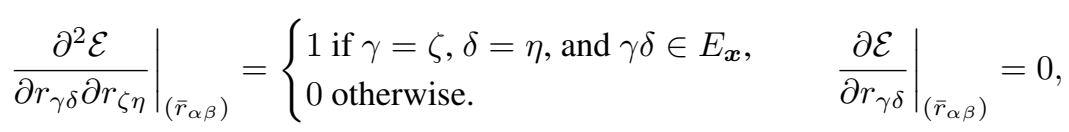

it follows that

$$
\mathbb{C}_{i j k l}^{*}=\frac{1}{V} \sum_{\alpha \beta \in E_{\boldsymbol{x}}} \frac{\overline{\boldsymbol{r}}_{\alpha \beta} \otimes \overline{\boldsymbol{r}}_{\alpha \beta} \otimes \overline{\boldsymbol{r}}_{\alpha \beta} \otimes \overline{\boldsymbol{r}}_{\alpha \beta}}{\bar{r}_{\alpha \beta}^{2}} .
$$

Comparing Eqn. (6.3) with Eqn. (6.5), it is clear that unlike $\mathbb{C}, \mathbb{C}^{*}$ is a geometric entity independent of the potential energy.

We now identify and motivate the following analogies between the atomistic entities defined in Section 4 and the continuum entities.

1. Local shape space and strain analogy:

$$
\begin{aligned}
& \begin{array}{l}
\text { Tangent space of the local } \\
\text { shape space }
\end{array} \Leftrightarrow\left\{\mathbb{C}^{*} \frac{\nabla \boldsymbol{u}+\nabla^{\mathrm{T}} \boldsymbol{u}}{2}: \boldsymbol{u} \in \mathcal{V}\right\} .
\end{aligned}
$$

A vector in the tangent space may be viewed as a compatible perturbation of distances. At the same time, it follows from the derivation of $\mathbb{C}^{*}$ that for a compatible strain field $\epsilon, \mathbb{C}^{*} \epsilon$ is a first-order approximation of the changes in the length of infinitesimal material bonds. This motivates the analogy given in Eqn. (6.6).

2. Atomistic and continuum stress analogy:

$$
\begin{array}{lll}
\boldsymbol{\sigma}_{w, \mathrm{v}}^{\|} & \Leftrightarrow & \boldsymbol{\sigma}_{\mathrm{c}}^{\|}, \\
\boldsymbol{\sigma}_{w, \mathrm{v}}^{\perp} & \Leftrightarrow & \boldsymbol{\sigma}_{\mathrm{c}}^{\perp} .
\end{array}
$$

Recall that $\boldsymbol{\sigma}_{w, \mathrm{v}}^{\|}$is defined using $\left(f_{\alpha \beta}^{\|}\right)$, which is the projection of $\left(f_{\alpha \beta}\right)$ onto the tangent space of $\mathcal{S}_{x}$ (see Theorem 1). At the same time, from Eqns. (5.15) and (5.12), and the 


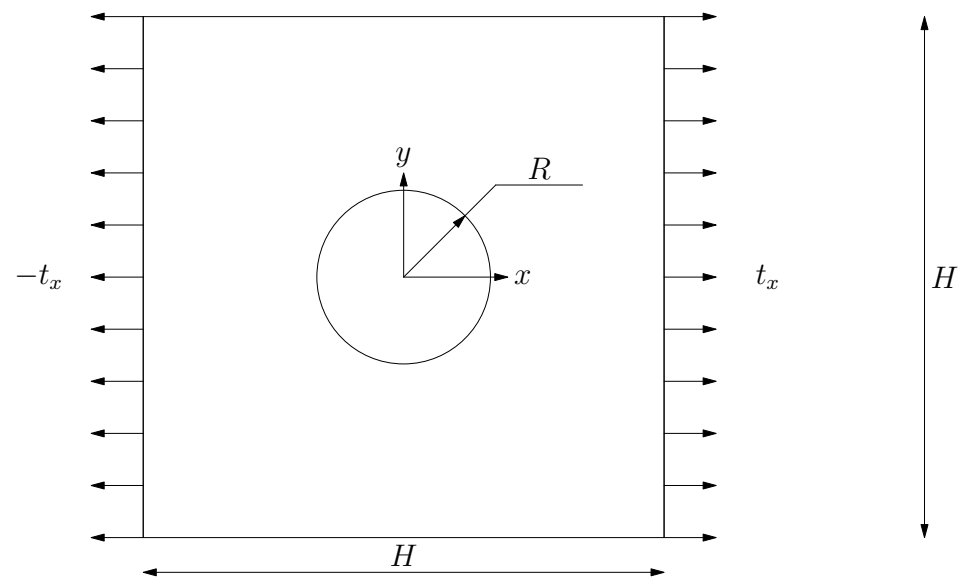

Figure 2: A schematic of the geometry of the studied boundary-value problem, with non-zero traction boundary conditions enforced on a part of the boundary as shown.

following identities,

$$
\operatorname{curl}\left(\operatorname{curl}\left(\nabla \boldsymbol{v}+\nabla \boldsymbol{v}^{\mathrm{T}}\right)\right) \equiv \mathbf{0}, \quad \operatorname{div}(\operatorname{curl}(\operatorname{curl} \boldsymbol{\Psi})) \equiv \mathbf{0},
$$

it follows that $\sigma_{\mathrm{c}}^{\|}$is the projection ${ }^{31}$ of $\sigma_{\mathrm{c}}$ onto the space defined on the right-hand-side of Eqn. (6.7). This motivates the analogies given in Eqns. (6.7) and (6.8).

3. It is important to note that the Beltrami decomposition of the continuum stress discussed in Section 5 depends on the choice of $\mathbb{C}^{\star}$. An analogous choice also exists in the decomposition of the atomistic stress tensor. This can be seen by noting that in order to arrive at the decomposition of the atomistic stress suggested in Section 4, we have implicitly assumed the Euclidean inner-product in the distance coordinate space. ${ }^{32}$ In this sense, the decomposition suggested in Section 4 depends on the choice of the inner-product. The Euclidean inner-product has resulted in a special choice of $\mathbb{C}^{\star}$ given in Eqn. (6.5). In other words, $\mathbb{C}^{\star}$ is directly related to the choice of the inner-product in the distance coordinate space.

In this paper, we do not subject the above analogies to further rigorous treatment. Instead, we check their validity using a numerical test in the next section.

\section{Numerical test}

In this section, we use a numerical example to compare the decompositions of the atomistic and continuum stress tensors given in Sections 4 and 5 respectively. We consider a rectangular anisotropic medium with a hole subjected to a uniaxial loading under plane strain conditions.

\footnotetext{
${ }^{31}$ The projection is with respect to the inner-product defined in Eqn. (5.14).

${ }^{32}$ The notion of perpendicularity and distance used in Eqn. (4.11) and Eqn. (4.29) respectively are defined with respect to the Euclidean inner-product.
} 
The orientation of the body and the boundary conditions are shown in Fig. 2. The size of the body, radius of the hole and the traction shown in Fig. 2 are chosen as

$$
H=300 \AA, \quad R=50 \AA, \quad \text { and } \quad \bar{t}_{x}=7.117 \times 10^{-5} \mathrm{eV} / \AA^{3},
$$

respectively. The body extends to infinity in the positive and negative $z$-directions. For the sake of comparison, the material is taken to be a single crystal Ar in the face-centered cubic (fcc) structure such that the $x, y$ and $z$ axes are oriented along the [100], [010] and [001] crystallographic directions. The system is studied at zero temperature.

In order to compare the decomposition of the continuum stress to that of the atomistic stress we consider two different models: (1) A linear elastic boundary-value problem, and (2) An atomistic model with a given potential energy $\mathcal{V}$. The elasticity tensor for the continuum model is obtained from the potential $\mathcal{V}$ of the atomistic model using Eqn. (6.3). In the next section, we describe the continuum boundary-value problem and the decomposition of the resulting continuum stress tensor.

\subsection{Decomposition of the continuum stress}

The continuum linear elastic constitutive law is given by

$$
\boldsymbol{\sigma}_{\mathrm{c}}=\mathbb{C} \frac{\nabla \boldsymbol{u}_{\mathrm{c}}+\nabla \boldsymbol{u}_{\mathrm{c}}^{\mathrm{T}}}{2},
$$

where $\boldsymbol{u}_{c}$ denotes a smooth displacement vector field on the domain $\Omega$. The continuum boundaryvalue problem is

$$
\begin{array}{rll}
\operatorname{div} \boldsymbol{\sigma}_{\mathrm{c}}(\boldsymbol{x})=\mathbf{0} & \text { for } & \boldsymbol{x} \in \Omega, \\
\boldsymbol{\sigma}_{\mathrm{c}}(\boldsymbol{x}) \boldsymbol{n}(\boldsymbol{x})=\boldsymbol{t}(\boldsymbol{x}) & \text { for } & \boldsymbol{x} \in \partial \Omega,
\end{array}
$$

where the traction $t$ on the boundary of $\Omega$ is shown in Fig. 2. For this numerical test, we assume that the loading at the external boundaries and the hole axes are parallel to the crystallographic axes of the plate. A numerical solution $\boldsymbol{u}_{\mathrm{c}}$ for the boundary-value problem Eqn. (7.3) is obtained using the finite element method (FEM) with linear triangular elements. The continuum stress $\sigma_{\mathrm{c}}$ can then be evaluated from $\boldsymbol{u}_{\mathrm{c}}$ using Eqn. (7.2). We now decompose $\boldsymbol{\sigma}_{\mathrm{c}}$ into $\boldsymbol{\sigma}_{\mathrm{c}}^{\|}$and $\boldsymbol{\sigma}_{\mathrm{c}}^{\perp}$.

Since $\sigma_{\mathrm{c}}^{\perp}$ is divergence-free and traction-free (see Eqn. (5.13)), it follows that $\sigma_{\mathrm{c}}^{\|}$also satisfies Eqn. (7.3). Moreover, since the expression for $\sigma_{\mathrm{c}}^{\|}$in Eqn. (5.13), is identical to the right-hand side of Eqn. (7.2) with $\mathbb{C}$ replaced with $\mathbb{C}^{*}, \sigma_{\mathrm{c}}^{\|}$can be obtained from the FEM implementation with material constants corresponding to $\mathbb{C}^{*}$ in Eqn. (6.5) for the equilibrium lattice configuration corresponding to the potential $\mathcal{V}$. Then $\sigma_{\mathrm{c}}^{\perp}$ follows as $\sigma_{\mathrm{c}}^{\perp}=\sigma_{\mathrm{c}}-\sigma_{\mathrm{c}}^{\|}$.

\subsection{Decomposition of the atomistic stress}

In the atomistic simulation, the boundary-value problem discussed above is modeled using a collection of Ar atoms in the fcc structure at zero temperature $(T=0 \mathrm{~K})$. The atoms interact according to a modified Lennard-Jones (MLJ) pair potential ${ }^{33} \mathcal{V}_{\mathrm{MLJ}}$ with parameters from

\footnotetext{
${ }^{33}$ In a modified LJ potential, the standard LJ potential is modified by the addition of a quadratic function to bring the potential and its first derivative to zero at the cutoff radius.
} 
Bernardes (1958) archived in OpenKIM (Admal, 2015; Tadmor et al., 2011). The LJ parameters are $\epsilon=10.4 \mathrm{meV}$ and $\sigma=3.4 \AA$. The cutoff radius is $r_{\text {cut }}=8.5 \AA$. The resulting equilibrium fcc lattice parameter at zero temperature is $a_{0}=5.29216 \AA$. The reference (unloaded) configuration of the plate is obtained by stacking $56 \times 56 \times 1$ unit cells with lattice parameter $a_{0}$, and removing all atoms that fall inside a circle of radius $50 \AA$ positioned at the center of the stack, with its out-of-plane axis parallel to the [001] crystallographic axis. The resulting system consists of 11652 atoms. The system is loaded by displacing the atoms according to the numerical solution $\boldsymbol{u}_{\mathrm{c}}$ of the boundary-value problem corresponding to $\mathbb{C}$ described in Section 7.1 and keeping them fixed at these positions. ${ }^{34}$

The atomistic stress field $\boldsymbol{\sigma}_{w, \mathrm{v}}$ is obtained using the expression given in Eqn. (2.13). The forces $f_{\alpha \beta}$ appearing in this expression are given by

$$
\boldsymbol{f}_{\alpha \beta}=-\frac{\partial \mathcal{V}}{\partial r_{\alpha \beta}} \frac{\boldsymbol{x}_{\alpha}-\boldsymbol{x}_{\beta}}{r_{\alpha \beta}},
$$

where $r_{\alpha \beta}=\left\|\boldsymbol{x}_{\alpha}-\boldsymbol{x}_{\beta}\right\|$, and $\boldsymbol{x}_{\alpha}$ and $\boldsymbol{x}_{\beta}$ are the positions of atoms $\alpha$ and $\beta$ at the end of the atomistic simulation. ${ }^{35}$ The weighting function in Eqn. (2.13) is taken to be a constant with a trigonometric mollifying function:

$$
w(\boldsymbol{r})=\widehat{w}(\|\boldsymbol{r}\|)= \begin{cases}c_{R} & \text { if }\|\boldsymbol{r}\|<R-\epsilon, \\ \frac{1}{2} c_{R}\left[1-\cos \left(\frac{R-\|\boldsymbol{r}\|}{\epsilon} \pi\right)\right] & \text { if } R-\epsilon<\|\boldsymbol{r}\|<R, \\ 0 & \text { otherwise, }\end{cases}
$$

where $c_{R}$ is chosen such that $w$ is a normalized function. We set $R=12 \AA$ and $\epsilon=1.44 \AA$. The atomistic stress tensor can now be decomposed using the algorithm described in Section 4.2.

\subsection{Comparison of the continuum and atomistic decompositions}

In this section, we compare the decompositions of the atomistic and continuum stress tensors for a given potential $\mathcal{V}$. The independent components of the elasticity tensor $\mathbb{C}$ calculated using Eqn. (6.3) for the interatomic potential $\mathcal{V}_{\mathrm{MLJ}}$ defined in Section 7.2 are

$$
\overline{\mathbb{C}}_{11}=0.02319 \mathrm{eV} / \AA^{3}, \quad \overline{\mathbb{C}}_{12}=\overline{\mathbb{C}}_{44}=0.01333 \mathrm{eV} / \AA^{3},
$$

where $\overline{\mathbb{C}}$ is used to denote the matrix form of $\mathbb{C}$ in Voigt notation. (Note that $\mathbb{C}$ has only two independent components due to the Cauchy relations for a pair potential.) Next, the independent components of $\mathbb{C}^{*}$ calculated using Eqn. (6.5) for a (cubic) fcc lattice structure with lattice constant $a_{0}=5.29216 \AA$ are

$$
\overline{\mathbb{C}}_{11}^{*}=22.22155 \mathrm{eV} / \AA^{3}, \quad \overline{\mathbb{C}}_{12}^{*}=\overline{\mathbb{C}}_{44}^{*}=5.5176 \mathrm{eV} / \AA^{3} .
$$

\footnotetext{
${ }^{34}$ In order to study the system in the absence of surface relaxation, we have not allowed the system to relax in the presence of surface tractions. We noted that that this assumption does not alter the stress profile, and its decomposition, in any appreciable manner in the bulk.

${ }^{35}$ Note that we carry out the atomistic decomposition on the deformed configuration, while the continuum decomposition described in Section 7.1 is on the fixed reference configuration. Since the deformation is assumed to be small the comparison between the atomistic and continuum decompositions remains valid.
} 

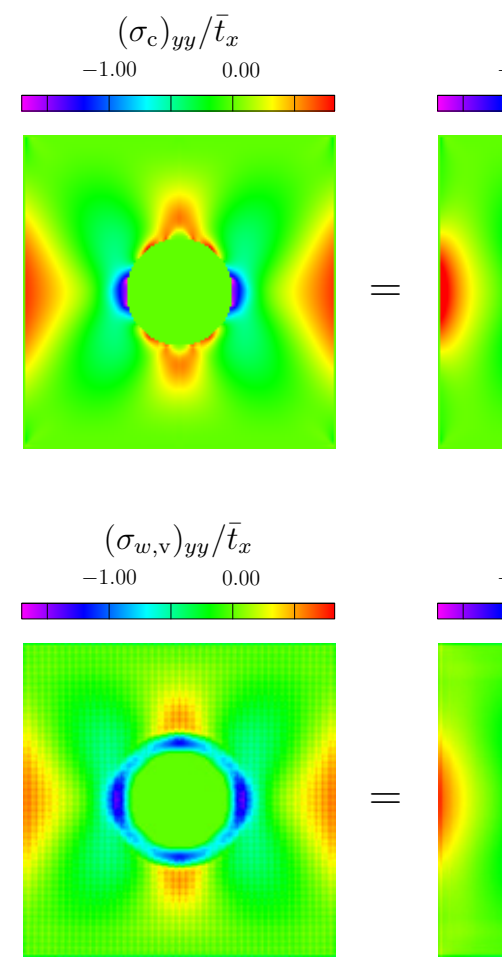
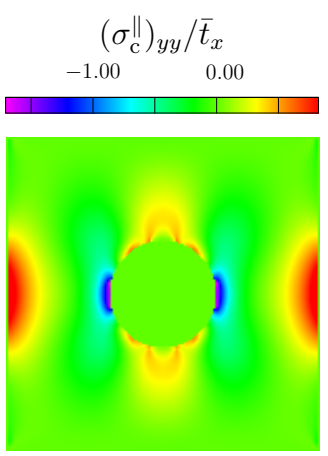

(a)

$\left(\sigma_{w, \mathrm{v}}^{\|}\right)_{y y} / \bar{t}_{x}$ $-1.00 \quad 0.00$

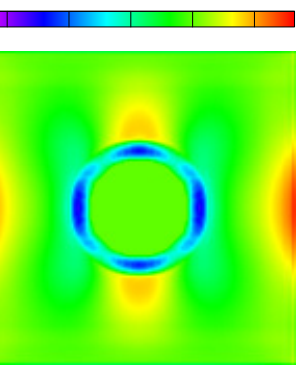

(b)
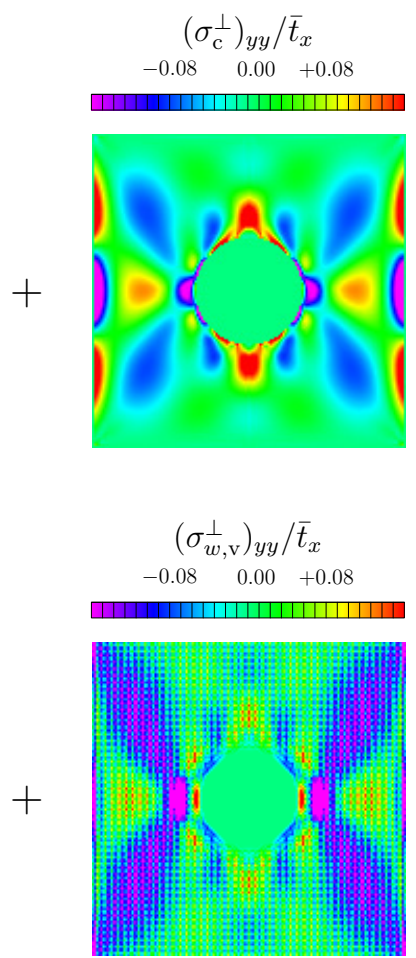

Figure 3: A plot showing the decomposition of the $y y$ component of (a) continuum stress, and (b) atomistic stress computed using the potential $\mathcal{V}_{\mathrm{MLJ}}$, into their respective irrotational and solenoidal parts. The stresses are normalized by the applied traction $\bar{t}_{x}$.

Using $a_{0}$ and the constants given in Eqns. (7.6) and (7.7), the continuum and the atomistic stress tensors can be constructed and decomposed as described in Section 7.1 and Section 7.2, respectively. The results for the $y y$-component of the stress normalized by the applied traction $\bar{t}_{x}$ are shown in Fig. 3. We observe that the decompositions are similar but not identical. The reason for this is the following fundamental difference between the atomistic stress and the continuum stress. Under zero loading, the continuum stress is zero and it decomposes trivially, i.e. $\sigma_{\mathrm{c}}^{\|}=\sigma_{\mathrm{c}}^{\perp}=\mathbf{0}$. In contrast, the atomistic stress field oscillates about zero, and has a non-trivial decomposition. One way to avoid this discrepancy is to alter the potential energy model in order to eliminate the difference between the continuum stress and the atomistic stress described 
$\left(\sigma_{\mathrm{c}}\right)_{x x} / \bar{t}_{x}$

$0.00+1.00+2.00 \quad+3.00$

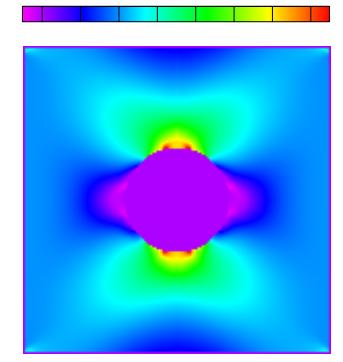

$\left(\sigma_{\mathrm{c}}\right)_{y y} / \bar{t}_{x}$

$-1.00 \quad 0.00$

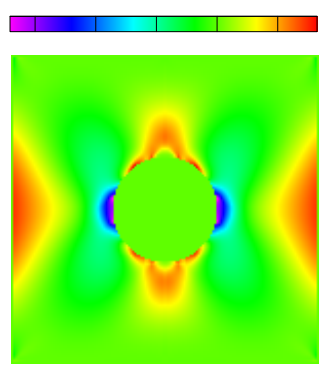

$\left(\sigma_{\mathrm{c}}\right)_{x y} / \bar{t}_{x}$

$\begin{array}{lll}-0.75 & 0.00 & +0.75\end{array}$
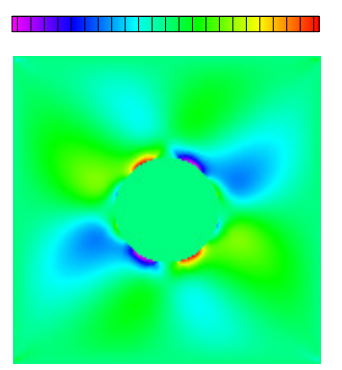

$\left(\sigma_{\mathrm{c}}^{\|}\right)_{x x} / \bar{t}_{x}$

$\begin{array}{llll}0.00 & +1.00 \quad+2.00 \quad+3.00\end{array}$

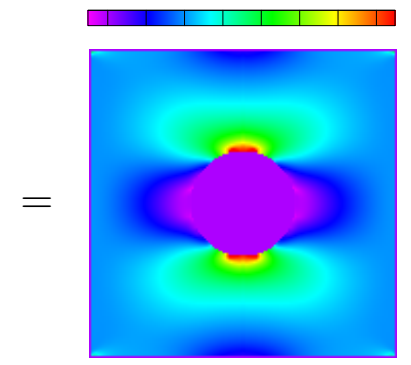

(a)

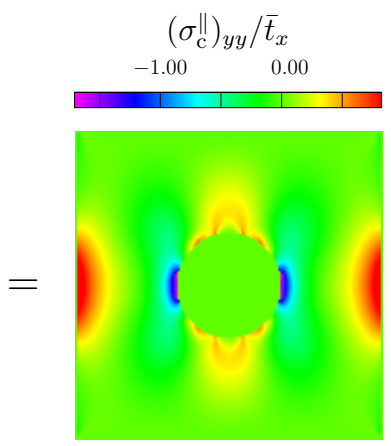

(b)

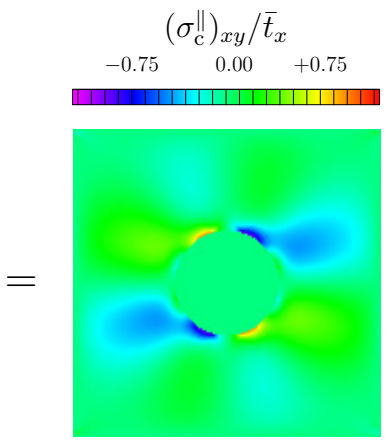

(c)
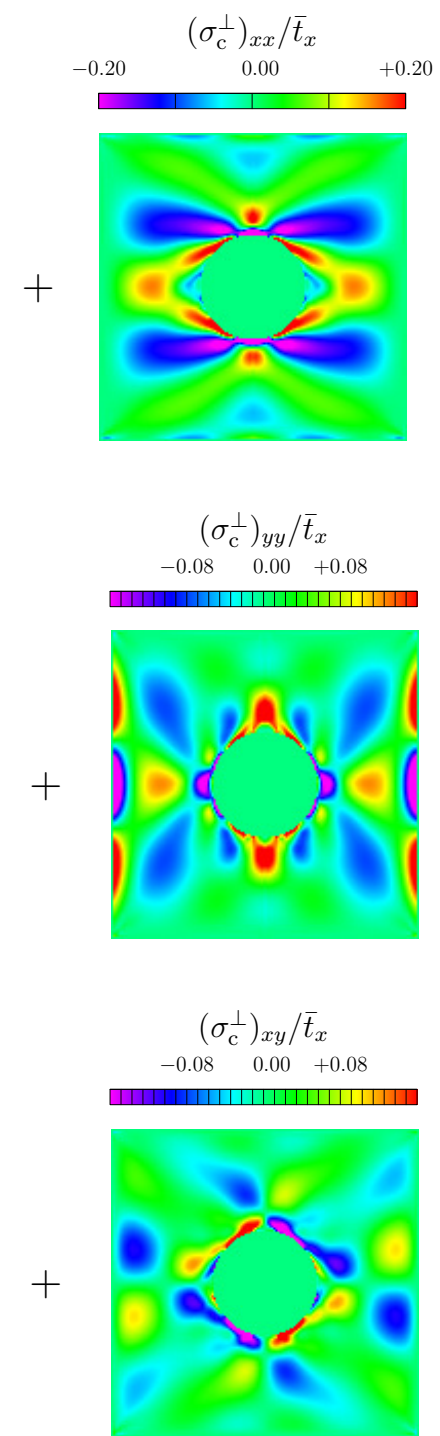

Figure 4: A plot showing the decomposition of the continuum stress into an irrotational part $\sigma_{\mathrm{c}}^{\|}$, and a traction-free solenoidal part $\sigma_{\mathrm{c}}^{\perp}$. Parts (a), (b), and (c) show the decomposition of the $x x, y y$ and $x y$ components of $\sigma_{\mathrm{c}}$, respectively. The stresses are normalized by the applied traction $\bar{t}_{x}$.

above. ${ }^{36}$ This is achieved by considering a new pair potential $\mathcal{V}_{\mathrm{S}}$ defined as

$$
\mathcal{V}_{\mathrm{s}}\left(\left(r_{\alpha \beta}\right)\right)=\frac{1}{2} \sum_{\substack{\alpha, \beta \\ \alpha<\beta}} \sum_{\substack{\gamma<\delta \\ \gamma<\delta}} k_{\alpha \beta \gamma \delta}\left(r_{\alpha \beta}-\bar{r}_{\alpha \beta}\right)\left(r_{\gamma \delta}-\bar{r}_{\gamma \delta}\right),
$$

\footnotetext{
${ }^{36}$ Another way to avoid this discrepancy is instead of comparing with the continuum stress, to compare two decompositions of the atomistic stress tensor $\boldsymbol{\sigma}_{w, \mathrm{v}}$ : (1) Using the algorithm described in Section 4, and (2) Using the algorithm discussed in Section 7.1, i.e. solve the boundary-value problem in Eqn. (7.3) with tractions obtained from $\boldsymbol{\sigma}_{w, \mathrm{v}}$. While
} 

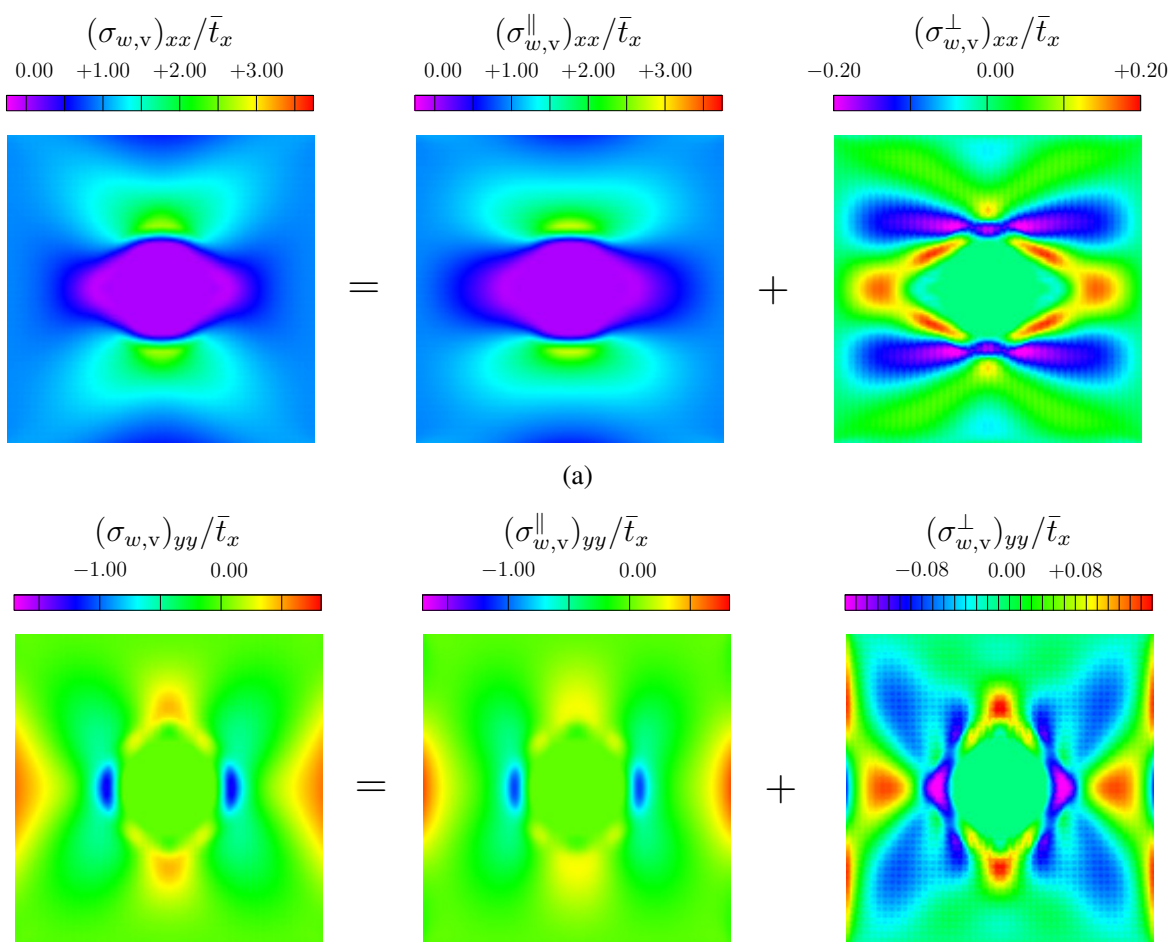

(b)
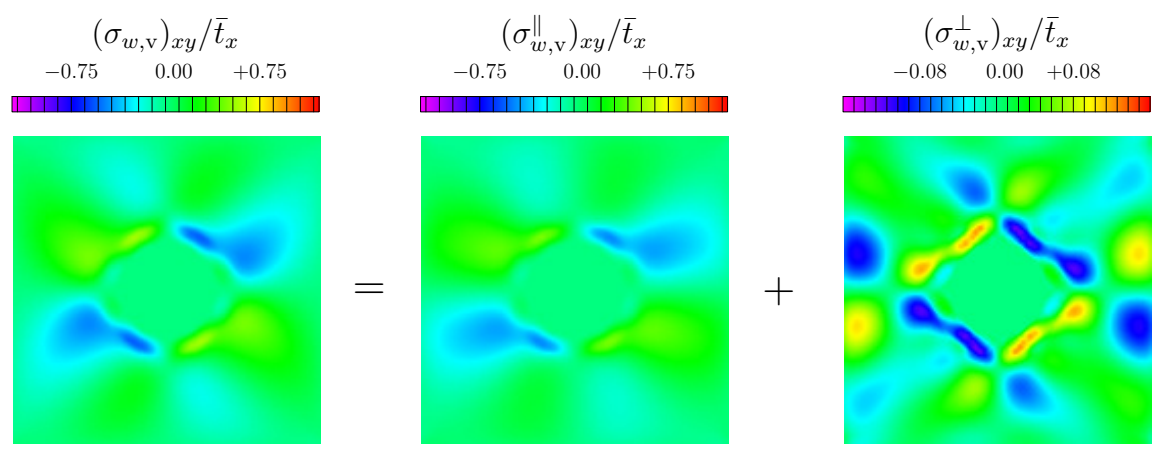

(c)

Figure 5: A plot showing the decomposition of the atomistic stress computed using the potential $\mathcal{V}_{\mathrm{s}}$ into an irrotational part $\boldsymbol{\sigma}_{w, \mathrm{v}}^{\|}$and a solenoidal part $\boldsymbol{\sigma}_{w, \mathrm{v}}^{\perp}$. Parts (a), (b), and (c) show the decomposition of the $x x$, $y y$ and $x y$ components of $\boldsymbol{\sigma}_{w, \mathrm{v}}$, respectively. The stresses are normalized by the applied traction $\bar{t}_{x}$.

this approach is the most direct and transparent way to compare the two decompositions, we do not take this approach because there is no unique way to obtain continuum tractions on a surface from an atomistic stress. 
where $\bar{r}_{\alpha \beta}$ is the distance between particles $\alpha$ and $\beta$ in the equilibrium fcc lattice at zero temperature corresponding to $\mathcal{V}_{\mathrm{MLJ}}$, and the spring constant $k_{\alpha \beta \gamma \delta}$ is obtained from $\mathcal{V}_{\mathrm{MLJ}}$ as

$$
k_{\alpha \beta \gamma \delta}=\left\{\begin{array}{l}
\left.\left(\frac{\partial^{2} \mathcal{V}_{M L J}}{\partial r_{\alpha \beta}^{2}}-\frac{1}{r_{\alpha \beta}} \frac{\partial \mathcal{V}_{M L J}}{\partial r_{\alpha \beta}}\right)\right|_{\left(\bar{r}_{\alpha \beta}\right)}, \text { if } \alpha=\gamma \text { and } \beta=\delta, \\
\left.\frac{\partial^{2} \mathcal{V}_{M L J}}{\partial r_{\alpha \beta} \partial r_{\gamma \delta}}\right|_{\left(\bar{r}_{\alpha \beta}\right)}, \text { otherwise. }
\end{array}\right.
$$

From the construction of $\mathcal{V}_{\mathrm{s}}$ in Eqn. (7.8), it is clear that the equilibrium lattice parameter and the elastic constants of $\mathcal{V}_{\mathrm{s}}$ remain the same as that obtained from $\mathcal{V}_{\text {MLJ }}$. Moreover, similar to the continuum stress, the atomistic stress resulting from $\mathcal{V}_{\mathrm{s}}$ is identically zero at zero loading. We now compare the decomposition of the atomistic and continuum stress tensors resulting from $\mathcal{V}_{\mathrm{s}}$. Figs. 4 and 5 show for the continuum and atomistic stresses, respectively, the decomposition of the $x x, y y$ and $x y$ components into irrotational and traction-free solenoidal parts. Comparing these figures (and referring back to Fig. 3), it is clear that after altering $\mathcal{V}_{\mathrm{MLJ}}$ to $\mathcal{V}_{\mathrm{s}}$, the decompositions are in very good agreement with each other. This suggests that the decomposition of the atomistic stress proposed in Section 4 is the correct analogue of the decomposition of the continuum stress obtained using the Beltrami representation in Section 5.

From Figs. 3(b) and 5(b), we observe that a large proportion of noise in the atomistic stress exists in the solenoidal part, while a large proportion of its macroscopic features exist in the irrotational part. Thus, there is a clear separation of scales that can be attributed to the decomposition of the atomistic stress tensor proposed in this paper. This suggests a new way for reducing noise in atomistic stress calculations. Current methods for noise reduction are based on the idea of selecting an optimal support for the weighting function that reduces noise without losing macroscopic features (Ulz et al., 2013). Our results indicate that a more effective noise reduction can be obtained by selecting two weighting functions with different-sized supports to obtain the atomistic stress. A weighting function with a larger support will be used to obtain the solenoidal part so that most of the noise is averaged out, and a weighting function with a smaller support will be used to obtain the irrotational part to best preserve the macroscopic features. This results in a systematic reduction of noise which may not be accessible using a single weighting function. This program of noise reduction will be pursued in a future work.

\section{Summary}

In this paper, we study the non-uniqueness of the atomistic stress tensor arising from the nonuniqueness of the force decomposition, which in turn is due to the choice of the potential energy representation. A geometric interpretation of force decomposition is given using rigidity theory and its non-uniqueness is characterized. This analysis results in a decomposition of the atomistic stress into an irrotational part that depends on the choice of potential energy representation, and a solenoidal part that is independent of it. A similar decomposition is constructed in continuum mechanics using the generalized Beltrami representation, which is a version of Helmholtz decomposition for symmetric tensor fields. We numerically compare the two decompositions and demonstrate their equivalence for a linear elasticity boundary-value problem of an anisotropic infinite plate with a hole under uniaxial stress at infinity. ${ }^{37}$ In addition, we observe that a large

\footnotetext{
${ }^{37}$ Since the numerical tests demonstrated in this paper are performed at a temperature of $0 \mathrm{~K}$, the kinetic part of the atomistic stress is zero and the potential part of the atomistic stress can be compared to the continuum Cauchy stress.
} 
proportion of the noise in the atomistic stress tensor is contained in the solenoidal part, while a large proportion of its macroscopic features is carried by the irrotational part. This feature of scale separation suggests a novel method for noise reduction in atomistic fields.

We have implemented Algorithm 1 discussed in Section 4.2 as a KIM-compliant Fortran program called MDStressLab, which was used to obtain the decomposition of the atomistic stress fields presented in Section 7. The code and a reference manual documenting its use are available online at http://mdstresslab.org/. Given the positions and velocities of all particles, and a KIM-compliant interatomic model (see https: / / openkim.org), MDStressLab calculates fields of the Cauchy and referential (Piola-Kirchhoff) versions of the virial, Tsai and the Hardy stress tensors, and their associated decompositions. ${ }^{38}$

\section{Acknowledgments}

This work was partly supported by the National Science Foundation under Awards No. PHY0941493 and DMR-1408211. Dr. Admal would like to gratefully acknowledge the support provided by the Institute for Pure and Applied Mathematics at the University of California Los Angeles where a part of this work was carried out. The authors would like to thank Amit Acharya for sowing the seed for this problem by suggesting a possible relationship between the decomposition of the atomistic stress and the Helmholtz decomposition for symmetric tensors. Additionally, the authors would like to thank Roger Fosdick, Richard D. James and Ryan S. Elliott for their valuable comments.

\section{References}

\section{References}

Admal, N. C., 2010. A unified interpretation of stress in molecular systems. Master's thesis, University of Minnesota, Department of Aerospace Engineering and Mechanics, Minneapolis, MN 55455.

Admal, N. C., 2015. Modified Lennard-Jones potential for Argon.

URL https: / / openkim.org/cite/MO_764178710049_000

Admal, N. C., Tadmor, E. B., 2010. A unified interpretation of stress in molecular systems. Journal of Elasticity 100 , 63-143.

Admal, N. C., Tadmor, E. B., 2011. Stress and heat flux for arbitrary multibody potentials: A unified framework. The Journal of Chemical Physics 134, 184106.

Admal, N. C., Tadmor, E. B., 2016. Material fields in atomistics as pull-backs of spatial distributions. Journal of the Mechanics and Physics of Solids 89, 59-76.

Beltrami, E., 1892. Osservazioni sulla nota precedente. Atti Accad. Lincei Rend 1 (5), 141-142.

Bernardes, N., 1958. Theory of solid Ne, Ar, Kr, and Xe at OK. Phys. Rev. 112 (5), 1534-1539.

For non-zero temperature, such a comparison can be carried out by first altering the atomistic stress to include the kinetic contribution. This alteration has been recently studied by the authors in Admal and Tadmor (2016) where the atomistic Cauchy stress tensor for a system under macroscopic equilibrium can be written using the average positions of the atoms in the reference configuration $\left(\boldsymbol{X}_{\alpha}\right)$ as

$$
\boldsymbol{\sigma}(\boldsymbol{X}, t)=\sum_{\substack{\alpha, \beta \\ \alpha<\beta}}-\boldsymbol{f}_{\alpha \beta} \otimes\left(\boldsymbol{X}_{\alpha}-\boldsymbol{X}_{\beta}\right) \int_{s=0}^{1} \delta\left((1-s) \boldsymbol{X}_{\alpha}+s \boldsymbol{X}_{\beta}-\boldsymbol{X}\right) d s .
$$

Using the decomposition of $\left(f_{\alpha \beta}\right)$ suggested in this paper, we can now arrive at the decomposition of the stress tensor, defined in Eqn. (8.1) for non-zero temperatures.

${ }^{38}$ See Admal and Tadmor (2016) for a rigorous definition of referential atomistic stress tensors. 
Connelly, R., Whiteley, W., 1996. Second-order rigidity and prestress stability for tensegrity frameworks. SIAM Journal on Discrete Mathematics 9 (3), 453-491.

Fosdick, R., Royer-Carfagni, G., 2005. A Stokes theorem for second-order tensor fields and its implications in continuum mechanics. International Journal of Non-Linear Mechanics 40 (2), 381-386.

Fosdick, R., Schuler, K., 2003. Generalized Airy stress functions. Meccanica 38 (5), 571-578.

Ghiloni, R., 2010. The Hodge decomposition theorem for general three-dimensional vector fields, without cuts. University of Trento.

Girault, V., Raviart, P.-A., 1979. Finite element approximation of the Navier-Stokes equations. Lecture Notes in Mathematics, Berlin Springer Verlag 749.

Gurtin, M., 1963. A generalization of the Beltrami stress functions in continuum mechanics. Archive for Rational Mechanics and Analysis 13 (1), 321-329.

Gurtin, M. E., 1962. On Helmholtz's theorem and the completeness of the Papkovich-Neuber stress functions for infinite domains. Archive for Rational Mechanics and Analysis 9 (1), 225-233.

Hardy, R. J., 1982. Formulas for determining local properties in molecular dynamics simulation: Shock waves. The Journal of Chemical Physics 76 (01), 622-628.

Hardy, R. J., Root, S., Swanson, D. R., 2002. Continuum properties from molecular simulations. AIP Conference Proceedings $620,363-366$.

Hauret, P., Kuhl, E., Ortiz, M., 2007. Diamond elements: a finite element/discrete-mechanics approximation scheme with guaranteed optimal convergence in incompressible elasticity. International Journal for Numerical Methods in Engineering 72 (3), 253-294.

Hörmander, L., 1990. The analysis of linear partial differential operators: Distribution theory and Fourier analysis. Springer Study Edition. Springer-Verlag.

Irving, J. H., Kirkwood, G., 1950. The statistical mechanics theory of transport processes. iv. the equations of hydrodynamics. The Journal of Chemical Physics 18 (06), 817-829.

Lee, J., 2012. Introduction to smooth manifolds. Vol. 218. Springer.

Maxwell, J. C., 1870. On reciprocal figures, frames and diagrams of forces. Translations of the Royal Society of Edinburgh XXVI, 1-43.

Maxwell, J. C., 1874. Van der waals on the continuity of the gaseous and liquid states. Nature 10, 477-80.

Murdoch, A. I., 1983. The motivation of continuum concepts and relations from discrete considerations. The Quarterly Journal of Mechanics and Applied Mathematics 36 (2), 163-187.

Murdoch, A. I., 2003. On the microscopic interpretation of stress and couple stress. Journal of Elasticity 71, 105-131.

Murdoch, A. I., 2007. A critique of atomistic definitions of the stress tensor. Journal of Elasticity 88, 113-140.

Murdoch, A. I., 2012. Physical Foundations of Continuum Mechanics. Cambridge University Press, New York.

Noll, W., 1955. Die herleitung der grundgleichungen der thermomechanik der kontinua aus der statistichen mechanik Journal of Rational Mechanics and Analysis 4, 627-646.

Paige, C. C., Saunders, M. A., 1982. LSQR: An algorithm for sparse linear equations and sparse least squares. ACM Transactions on Mathematical Software (TOMS) 8 (1), 43-71.

Renardy, M., Rogers, R. C., 2004. An introduction to partial differential equations. Vol. 4. Springer

Tadmor, E. B., Elliott, R. S., Phillpot, S. R., Sinnott, S. B., 2013. NSF cyberinfrastructures: A new paradigm for advancing materials simulation. Current Opinion in Solid State and Materials Science 17 (6), 298-304.

Tadmor, E. B., Elliott, R. S., Sethna, J. P., Miller, R. E., Becker, C. A., 2011. The potential of atomistic simulations and the Knowledgebase of Interatomic Models. JOM Journal of the Minerals, Metals and Materials Society 63 (7), 17-17.

Tadmor, E. B., Miller, R. E., 2011. Modeling Materials: Continuum, Atomistic and Multiscale Techniques. Cambridge University Press.

Tadmor, E. B., Miller, R. E., Elliott, R. S., 2012. Continuum Mechanics and Thermodynamics: From Fundamental Concepts to Governing Equations. Cambridge University Press.

Tsai, D. H., 1979. The virial theorem and stress calculation in molecular dynamics. The Journal of Chemical Physics 70 (03), 1375-1382.

Ulz, M. H., Mandadapu, K. K., Papadopoulos, P., 2013. On the estimation of spatial averaging volume for determining stress using atomistic methods. Modelling and Simulation in Materials Science and Engineering 21 (1), 15010-15024.

Vanegas, J. M., Torres-Sánchez, A., Arroyo, M., 2014. Importance of force decomposition for local stress calculations in biomembrane molecular simulations. Journal of Chemical Theory and Computation 10 (2), 691-702.

\section{Appendix A. Periodic boundary conditions}

In this section, we discuss atomistic systems under orthogonal periodic boundary conditions, and construct the rigidity map and the associated Jacobian in order to decompose the atomistic 
stress tensor. Under periodic boundary conditions, the configuration of a system of $N$ interacting particles enclosed in an orthogonal box of dimensions $L_{1}, L_{2}$ and $L_{3}$ is described by $\boldsymbol{x}=\left(\boldsymbol{x}_{1}, \ldots, \boldsymbol{x}_{N}, \boldsymbol{L}\right) \in \mathbb{R}^{3 N+3}$, where $\boldsymbol{L}=\left(L_{1}, L_{2}, L_{3}\right)$. We assume that the edges of the orthogonal box are oriented along the coordinate axes. In addition, we adopt the minimum image convention, $L_{i}>2 r_{\text {cut }}(i=1,2,3)$, so that a particle $\alpha$ interacts with a particle $\beta$ or the image of $\beta$, but not both. The set of local edges $E_{\boldsymbol{x}}$ defined in Eqn. (3.4) is now given by

$$
E_{\boldsymbol{x}}:=\left\{\alpha \beta:\left\|\left(\boldsymbol{x}_{\alpha}-\boldsymbol{x}_{\beta}\right)^{\#}\right\|<r_{\text {cut }}\right\},
$$

where for any $\boldsymbol{y} \in \mathbb{R}^{3}, y_{i}^{\#}:=\left(L_{i} / 2+y_{i}\right) \bmod L_{i}-L_{i} / 2, i=1,2,3$. Similar to the discussion in Section 3, it can be easily shown that a local configuration space $\mathcal{N}_{\boldsymbol{x}}$ exists as a neighborhood of $\boldsymbol{x}$ in $\mathbb{R}^{3 N+3}$ such that for every $\boldsymbol{y} \in \mathcal{N}_{\boldsymbol{x}}, E_{\boldsymbol{y}}=E_{\boldsymbol{x}}$. The local rigidity map $R: \mathcal{N}_{\boldsymbol{x}} \rightarrow \mathbb{R}^{e}$, where $e$ is the cardinality of $E_{\boldsymbol{x}}$ is defined as

$$
\boldsymbol{R}(\boldsymbol{y})=\left(r_{E_{\boldsymbol{x}}(1)}, \ldots, r_{E_{\boldsymbol{x}}(e)}\right), \quad \boldsymbol{y} \in \mathcal{N}_{\boldsymbol{x}},
$$

with $r_{E_{\boldsymbol{x}}}(k)=\left\|\left(\boldsymbol{y}_{\alpha}-\boldsymbol{y}_{\beta}\right) \#\right\|$, where $E_{\boldsymbol{x}}(k)=\alpha \beta$. The local shape space $\mathcal{S}_{\boldsymbol{x}}$ is defined as the image of the local rigidity map, i.e. $\mathcal{S}_{\boldsymbol{x}}:=\operatorname{Im} \boldsymbol{R}$. The Jacobian associated with the rigidity map for periodic boundary conditions is an $e \times(3 N+3)$ matrix given by

$$
\left(\mathfrak{R}_{\boldsymbol{x}}\right)_{i j}= \begin{cases}\left(\boldsymbol{x}_{\alpha}-\boldsymbol{x}_{\beta}\right)_{k}^{\#} / r_{\alpha \beta}^{\#}, & j \in\{3(\alpha-1)+k: k=1,2,3\}, \\ \left(\boldsymbol{x}_{\beta}-\boldsymbol{x}_{\alpha}\right)_{k}^{\#} / r_{\alpha \beta}^{\#}, & j \in\{3(\beta-1)+k: k=1,2,3\}, \\ \left|\left(\boldsymbol{x}_{\alpha}-\boldsymbol{x}_{\beta}\right)_{k}^{\#}\right| / r_{\alpha \beta}^{\#}, & j \in\{3 N+1,3 N+2,3 N+3\} \text { and } r_{\alpha \beta}^{\#} \neq r_{\alpha \beta}, \\ 0, & \text { otherwise. }\end{cases}
$$

Theorem 1 and Corollaries 1, 2, and 3 discussed in Section 4 all remain valid for periodic boundary conditions using the Rigidity map and its Jacobian given in Eqns. (A.2) and (A.3) respectively. 